\title{
Lista atualizada da flora vascular do Parque Nacional (PARNA) Serra de Itabaiana, Sergipe, Brasil
}

\author{
Ana Cecília da Cruz Silva ${ }^{1}$ (D), Eduardo Vinícius da Silva Oliveira ${ }^{1}$ (D), Marccus \\ Alves $^{2}$ (D), Marta Cristina Vieira Farias ${ }^{1}$, Aline da Costa Mota ${ }^{3}$, Christopher \\ Anderson Santos Souza ${ }^{1} \&$ Ana Paula do Nascimento Prata ${ }^{4}$ (D)
}

(1) Universidade Federal de Sergipe, Centro de Ciências Biológicas e da Saúde, Departamento de Biologia, Cidade Universitária Prof. José Aloísio de Campos, Av. Marechal Rondon, Jardim Rosa Elze, São Cristóvão 49100-000, Sergipe, Brasil. E-mail: ceciliabio83@gmail.com, eduardovso@yahoo.com.br

(2) Universidade Federal de Pernambuco, Centro de Ciências Biológicas, Departamento de Botânica, Av. Moraes Rego, Cidade Universitária 50740-465, Recife, Pernambuco, Brasil. E-mail: alves.marccus@gmail.com

(3) Universidade de Pernambuco, Colegiado de Ciências Biológicas, BR 203, Km 2, Vila Eduardo, Petrolina, PE, Brasil. E-mail: alinecostamota@gmail.com

(4) Universidade Federal de Alagoas, Centro de Ciências Agrárias, BR 104 Norte, Km 85, Mata do Rolo, Rio Largo 57100-000, Alagoas, Brasil. E-mail: ana.prata@ceca.ufal.br

Silva A.C.C., Oliveira E.V.S., Alves M., Farias M.C.V., Mota A.C., Souza C.A.S. \& Prata A.P.N. (2019) Lista atualizada da flora vascular do Parque Nacional (PARNA) Serra de Itabaiana, Sergipe, Brasil. Pesquisa e Ensino em Ciências Exatas e da Natureza, 3(1): 40-67. http://dx.doi.org/10.29215/pecen.v3i1.1148

Editor acadêmico: Francisco Carlos P. da Costa. Recebido: 26 Outubro 2018. Aceito: 21 Fevereiro 2019. Publicado: 27 Maio 2019.

Resumo: O Parque Nacional Serra de Itabaiana está inserido numa região de ecótono entre os domínios fitogeográficos Mata Atlântica e Caatinga. Por ser uma das principais áreas de estudos científicos em Sergipe, existem trabalhos que abordam famílias botânicas e listagens da sua flora vascular. Entretanto, percebeu-se a necessidade de atualização e compilação das listas para melhor conhecimento da diversidade e conservação da flora local. Diante disso, elaborou-se um checklist a partir de publicações pretéritas e do banco de dados do Herbário da Universidade Federal de Sergipe (ASE). Foram registradas 124 famílias e 830 espécies, distribuídas entre Angiospermas (803 espécies), Samambaias e Licófitas (26) e Gimnospermas (1). As famílias de maior riqueza foram: Fabaceae (73 espécies), Poaceae (65), Cyperaceae (59), Rubiaceae (40) e Asteraceae (36). Ervas (41.2\%), arbustos (24.7\%) e árvores (17.6\%) foram os hábitos mais representativos. Cento e setenta espécies são novas ocorrências para Sergipe, há uma nova espécie para a ciência, seis espécies raras, três em perigo de extinção, nove quase ameaçadas e três vulneráveis, além de 80 espécies endêmicas da Mata Atlântica e 13 endêmicas da Caatinga.

Palavras chave: Checklist, coleção biológica, ecótono, florística.

\section{Current list of vascular flora of the Serra de Itabaiana National Park (PARNA), Sergipe, Brazil}

Abstract: The Serra de Itabaiana National Park is located in an ecotone between Atlantic Rainforest and Caatinga biomes. It is one of the main areas of scientific studies in Sergipe. There are works approaching botanical families and listings of vascular flora. However, there is a need to update and compile the lists to better knowledge of the diversity of local flora. Therefore, a checklist was prepared from previous publications and Herbarium database of the Federal University of Sergipe (ASE). In total 124 families and 830 species of vascular plants were recorded distributed between Angiosperm (803 species), Ferns and Lycophytes (26) and Gymnosperms (1). The families of greater richness were: Fabaceae (73 species), Poaceae (65), Cyperaceae (59), Rubiaceae (40) and Asteraceae (36). The most representative habits were herbs $(41.2 \%)$, shrubs $(24.7 \%)$ and tree $(17.6 \%)$. One hundred and seventy species are new occurrences for Sergipe, there is a new species for science, six rare species, three endangered species, nine almost 
endangered and three vulnerable, in addition there are 80 endemic species of the Atlantic Forest and 13 endemic to the Caatinga.

Key words: Biological collection, checklist, ecotone, floristic.

\section{Introdução}

Um dos vários mecanismos estabelecidos para preservar a biodiversidade brasileira é a implantação de Unidades de Conservação. Parque Nacional é uma das categorias de proteção integral definidas pelo SNUG (Brasil 2000). Em Sergipe, o Parque Nacional (PARNA) Serra de Itabaiana foi criado em 2005 com o objetivo de preservar os ecossistemas, possibilitar a realização de pesquisas científicas e o desenvolvimento de atividades de educação ambiental e turismo ecológico (Brasil 2005).

Esse Parque apresenta características únicas por situar-se em uma zona de transição onde coexistem espécies da Mata Atlântica e da Caatinga (Vicente et al. 2005). Por estar situada em zona de transição climática e em altitude mais elevada existem diferentes tipos vegetacionais (Dantas et al. 2010). Além disso, abriga uma fitofisionomia peculiar por estar assentada sobre as Areias Brancas, considerada um refúgio ecológico devido as condições específicas na formação do substrato (Dantas et al. 2010). Por essas razões, essa área foi considerada prioritária para conservação biológica (MMA 2007). Apesar de suas peculiaridades, o PARNA Serra de Itabaiana sofre pressão antrópica, como o uso recreativo de trilhas sem controle de visitação (Oliveira 2008). Também são comuns a retirada de madeira e areia, caça, queimadas, práticas agrícolas, depósito de lixo e introdução de espécies exóticas (Sobral et al. 2007).

Mesmo com o considerável nível de informações sobre a biodiversidade brasileira, ainda existem muitas lacunas que necessitam ser preenchidas, inclusive para colaborarem com ações de conservação (Brandon et al. 2005). Assim, a elaboração de listas florísticas é imprescindível, uma vez que muitas espécies são extintas localmente antes mesmo de terem sido registradas (Pereira et al. 2011), especialmente em áreas classificadas como prioritárias para a conservação (Giulietti et al. 2005). Contudo, atualmente há uma redução no número de taxonomistas em instituições, causada pela diminuição do apoio financeiro e científico, o que ocasiona uma diminuição nas descobertas de novas espécies (Christenhusz \& Byng 2016).

Por ser uma das principais áreas de estudos científicos em Sergipe, existem alguns trabalhos de famílias botânicas e listagens da flora vascular, como em: Vicente (1999), Vicente et al. (2005), Dantas et al. (2010), Dantas \& Ribeiro (2010), Maciel \& Alves (2011) e Pessoa \& Alves (2011). Entretanto, percebeu-se a necessidade de atualização e compilação de listas para melhor conhecimento da diversidade da flora local. Com o desenvolvimento do Projeto "Flora de Sergipe”, vários especialistas corrigiram e atualizaram o nome científico dos táxons. Diante do exposto, este trabalho foi realizado com o objetivo de gerar um checklist das espécies de plantas vasculares do PARNA Serra de Itabaiana, visando contribuir para o conhecimento e conservação da flora de Sergipe.

\section{Material e Métodos}

O Parque Nacional (PARNA) Serra de Itabaina abrange quase 8.000 ha e está localizado na região central de Sergipe $\left(10^{\circ} 40^{\prime} \mathrm{S}, 37^{\circ} 25^{\prime} \mathrm{O}\right)$, cerca de $40 \mathrm{~km}$ do litoral (Figura 1). Ocupa parte dos municípios de Areia Branca, Campo do Brito, Itabaiana, Itaporanga d'Ajuda e Laranjeiras, com a maioria de sua área estabelecida sobre o conjunto de serras residuais que compõem o Domo esvaziado de Itabaiana: Serra de Itabaiana, Serra do Cajueiro, Serra Comprida e Serra da Ribeira, com altitudes que variam entre 400 e 660 m (Santos \& Andrade 1992).

O clima predominante na região, segundo a Classificação de Köppen, é do tipo As, tropical, com verão seco e moderado excedente hídrico no inverno, com precipitações anuais entre 900 a $1200 \mathrm{~mm}$ (Seplan 1979). 
A vegetação é classificada como uma formação florestal ecotonal entre a Floresta Ombrófila Densa de Terras Baixas e Floresta Estacional Semidecídua Submontana (Dantas \& Ribeiro 2010). Dois tipos fitofisionômicos são encontrados (Vicente et al. 2005): áreas abertas com solos arenosos brancos e áreas fechadas com vegetação arbórea. Entretanto, são poucos os remanescentes contínuos de vegetação primária (Franco 1983; Vicente et al. 2005).
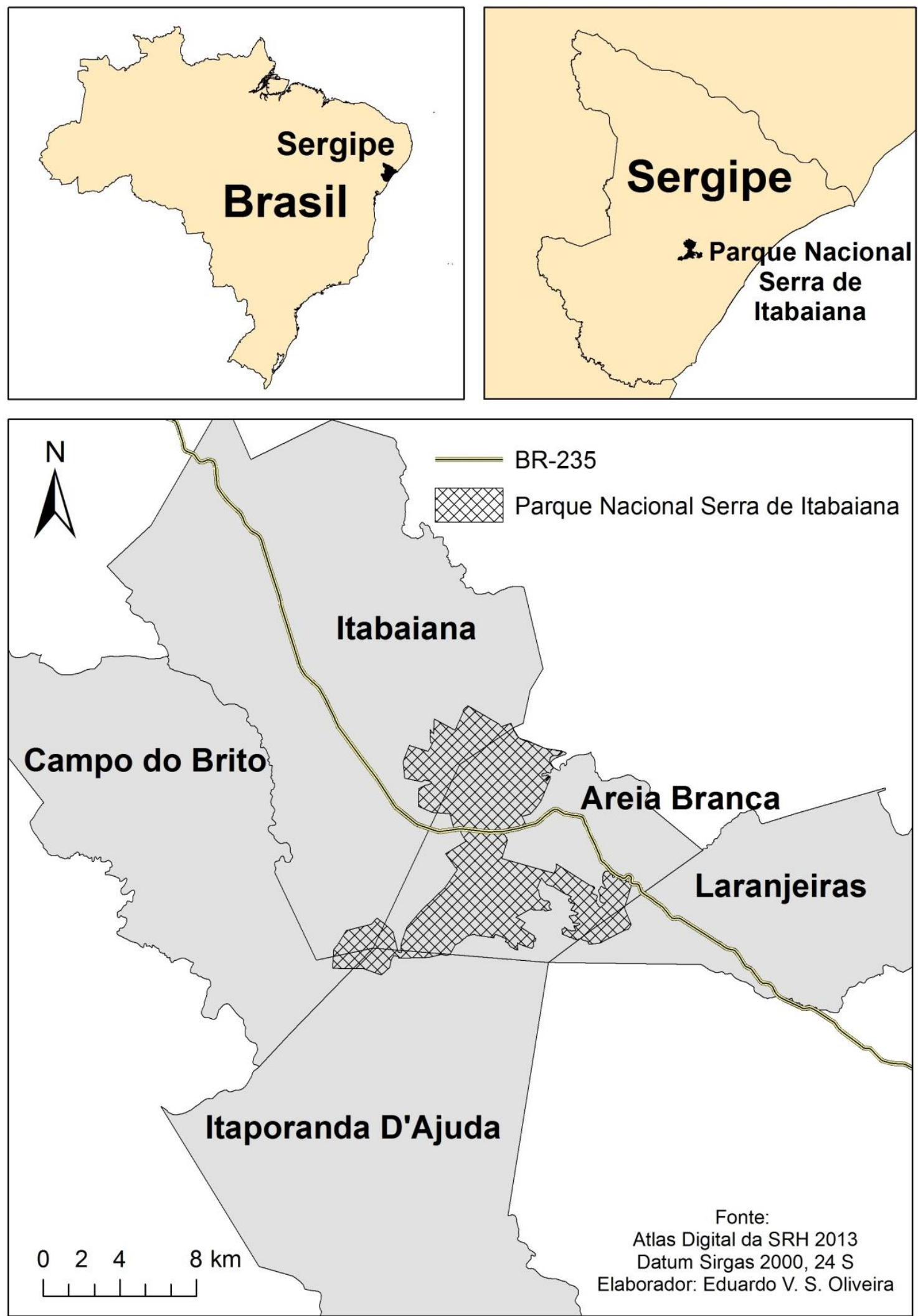

Figura 1. Localização da área de estudo, Parque Nacional Serra de Itabaiana, Sergipe, Brasil. 
Os dados desse estudo foram compilados de publicações (Vicente 1999; Dantas et al. 2010; Dantas \& Ribeiro 2010; Mendes et al. 2010; Maciel \& Alves 2011; Pessoa \& Alves 2011) e do banco de dados do Herbário da Universidade Federal de Sergipe (ASE), com auxílio do software "Botanical Research and Herbarium Management System (BRAHMS)", versão 7.1, buscando registros de espécies coletadas na área de estudo. Para as amostras em nível de gênero ou família foram mantidos apenas aqueles que representavam registros únicos para cada nível hierárquico. Registros oriundos das publicações específicas sobre o PARNA Serra de Itabaiana foram checados na plataforma SpeciesLink (http://splink.cria.org.br/tools) do Centro de Referência em Informação Ambiental (CRIA) para atualizações na identificação, informações do coletor e do herbário. Para os registros não encontrados nesta plataforma, utilizou-se a citação do artigo como referência na lista final de espécies.

A correção do banco de dados do Herbário ASE foi realizada com base nos dados disponibilizados na ferramenta dataCleaning, disponível na plataforma do SpeciesLink (http://splink.cria.org.br/dc/index?criaLANG=pt) do CRIA. Essa ferramenta faz o cruzamento de dados de todas as exsicatas e duplicatas distribuídas nos herbários que fazem parte da rede CRIA, incluindo a checagem de dados taxonômicos (grafia dos táxons), dados geográficos (principalmente coordenadas e nomes de cidades) e possíveis registros repetidos. Os principais dados analisados foram: georreferenciamento e dados taxonômicos. Foram utilizadas ainda as ferramentas geoLoc (também da plataforma SpeciesLink) e IBGE Cidades (disponível no sítio do

IBGE - http://www.cidades.ibge.gov.br/xtras/home.php?lang=_EN) no auxílio do georreferenciamento dos dados de parte da coleção. Ressalta-se que todas as espécies pertencentes a listagem e depositadas no Herbário ASE foram conferidas na coleção.

Foi adotado o sistema de classificação APG IV (2016), a nomenclatura dos táxons e as novas ocorrências para o Parque e para Sergipe foram verificadas no sítio da BFG (2018), com auxílio do pacote "flora" no aplicativo R versão 3.2.1 (R Development Core Team 2015; Carvalho 2017). A partir dessa lista florística, as plantas foram classificadas em raras (Giulietti et al. 2009), ameaçadas de extinção (BFG 2018) e endêmicas da Mata Atlântica e da Caatinga (BFG 2018).

\section{Resultados e Discussão}

A Flora do Parque Nacional Serra de Itabaiana está representada por 124 famílias e 828 espécies (Tabela 1; Figura 2), distribuídas entre Angiospermas (801 espécies), Samambaias e Licófitas (26) e Gimnospermas (1). Os números de espécies e de famílias foram bem superiores em relação a mais abrangente lista florística publicada, que foi a de Mendes et al. (2010), com 552 espécies e 100 famílias.

Com relação a Samambaias e Licófitas, a área estudada apresentou 65\% das 40 espécies registradas para Sergipe (BFG 2018). Essa elevada representatividade também foi observada por Pereira et al. (2011) em uma área de Floresta Atlântica em Pernambuco. Desse grupo, Polypodiaceae, considerada a terceira família mais representativa da Floresta Atlântica (Stehmann et al. 2009), predominou com oito espécies.

Podocarpus sellowii Klotzsch ex Endl. (Podocarpaceae) foi à única representante de Gimnospermas. Esta espécie é usualmente encontrada em locais de altitudes elevadas, como na floresta de brejo de Itatinga, em São Paulo (Ivanaukas et al. 1997). O gênero Podocarpus L'Hér. ex Pers. apresenta distribuição ecológica restrita e sua primeira citação no nordeste e na área de estudo foi feita por Andrade-Lima, em 1962 (Lima 2006). A população de $P$. sellowii nessa área é a mais criticamente ameaçada do Nordeste, com o número estimado de apenas cinco indivíduos (Falcão 2004). O último registro de depósito de material no Herbário ASE foi do ano de 1995. Entretanto, em 2009, Mendes et al. (2010) observaram uma grande população de indivíduos jovens nesse local e depositaram amostras no Herbário UFP - Geraldo Mariz, em Pernambuco. Ainda assim, em razão da baixa densidade populacional, $P$. sellowii pode ser considerada localmente rara (MMA 2003) e, portanto muito vulnerável à extinção local (Primack \& Rodrigues 2001). 
Tabela 1. Lista de plantas vasculares do Parque Nacional Serra de Itabaiana, Sergipe, Brasil. HAB: hábitos (Arb: arbusto; Arb-Epi: arbusto epífita; Arv: árvore; Erv: erva; Erv-Epi: erva epífita; Erv-Hpa: arbusto hemiparasita; Erv-Sap: erva saprófita; Pal: palmeira; Sub: Subarbusto; Tre-H: trepadeira herbácea; Tre-L: trepadeira lenhosa); OCO: ocorrências (NR: novo registro em Sergipe); CAT: categorias (DD: deficiente de dados; ECA: endêmica da Caatinga; EMA: endêmica da Mata Atlântica; EN: em perigo; NE: não avaliada quanto à ameaça de extinção; NT: quase ameaçada; LC: pouco preocupante; RAR: rara; VU: vulnerável).

\begin{tabular}{|c|c|c|c|c|}
\hline Família/Espécie & HAB & $\mathrm{OCO}$ & CAT & Coletor no./Autor(es) \\
\hline \multicolumn{5}{|c|}{ SAMAMBAIAS E LICÓFITAS } \\
\hline \multicolumn{5}{|l|}{ Anemiaceae } \\
\hline Anemia oblongifolia (Cav.) Sw. & Erv & - & $\mathrm{NE}$ & Viana, G 1852 (ASE) \\
\hline Anemia tomentosa (Sav.) Sw. & Erv & - & $\mathrm{NE}$ & Costa, SM 384 (ASE) \\
\hline \multicolumn{5}{|l|}{ Aspleniaceae } \\
\hline $\begin{array}{l}\text { Asplenium cristatum Lam. } \\
\text { Blechnaceae }\end{array}$ & \multicolumn{3}{|c|}{ Blechnaceae } & Silva, FO 277 (ASE) \\
\hline Blechnum occidentale $\mathrm{L}$. & Erv & - & $\mathrm{NE}$ & Santos, LAS 77 (ASE) \\
\hline Telmatoblechnum serrulatum (Rich.) & Erv & - & $\mathrm{NE}$ & Viana, G 1813 (ASE) \\
\hline \multicolumn{2}{|l|}{ Cyatheaceae } & & & \\
\hline Cyathea microdonta (Desv.) Domin & Arv & - & $\mathrm{NE}$ & Santiago, A 1312 (ASE) \\
\hline $\begin{array}{l}\text { Cyathea phalerata Mart. } \\
\text { Gleicheniaceae }\end{array}$ & \multicolumn{3}{|c|}{ Gleicheniaceae } & Silva, FO 175 (ASE) \\
\hline \multicolumn{5}{|l|}{ Hymenophyllaceae } \\
\hline Trichomanes cristatum Kaulf. & Erv & - & $\mathrm{NE}$ & Costa 375 (ASE) \\
\hline \multicolumn{5}{|l|}{ Lindsaeaceae } \\
\hline Lindsaea lancea (L.) Bedd. & Erv & - & $\mathrm{NE}$ & Santos, LAS 72 (ASE) \\
\hline $\begin{array}{l}\text { Lindsaea stricta (Sw.) Dryand. } \\
\text { Lomariopsidaceae }\end{array}$ & \multicolumn{3}{|c|}{ Lomariopsidaceae } & Costa 377 (ASE) \\
\hline $\begin{array}{l}\text { Nephrolepis biserrata (Sw.) Schott. } \\
\text { Lygodiaceae }\end{array}$ & \multicolumn{3}{|c|}{ Lygodiaceae } & Costa 450 (ASE) \\
\hline Lygodium venustum $\mathrm{Sw}$. & Tre-L & - & $\mathrm{NE}$ & Matos, ECA 35 (ASE) \\
\hline \multicolumn{5}{|l|}{ Polypodiaceae } \\
\hline Campyloneurum nitidum (Kaulf.) C.Presl & Erv-Epi & NR & $\mathrm{NE}$ & Santos, JL 16 (ASE) \\
\hline Campyloneurum repens (Aubl.) C. Presl & Erv & NR & NE & Landim 1035 (ASE) \\
\hline $\begin{array}{l}\text { Microgramma crispata (Fée) R.M.Tryon \& } \\
\text { A.F.Tryon }\end{array}$ & Erv-Epi & - & LC, EMA & Saka 220 (ASE) \\
\hline $\begin{array}{l}\text { Microgramma squamulosa (Kaulf.) de la } \\
\text { Sota }\end{array}$ & Erv-Epi & NR & $\mathrm{NE}$ & Silva, FO 253 (ASE) \\
\hline $\begin{array}{l}\text { Microgramma vacciniifolia (Langsd. \& } \\
\text { Fisch.) Copel. }\end{array}$ & Erv-Epi & - & $\mathrm{NE}$ & Santos, ACAS 92 (ASE) \\
\hline Pleopeltis astrolepis (Liebm.) E.Fourn. & Erv-Epi & NR & $\mathrm{NE}$ & Santos, JL 1 (ASE) \\
\hline $\begin{array}{l}\text { Pleopeltis macrocarpa (Bory ex Willd.) } \\
\text { Kaulf. }\end{array}$ & Erv-Epi & NR & $\mathrm{NE}$ & Santos, JL 33 (ASE) \\
\hline $\begin{array}{l}\text { Serpocaulon triseriale (Sw.) A.R.Sm. } \\
\text { Pteridaceae }\end{array}$ & Erv-Epi & NR & $\mathrm{NE}$ & Nascimento-Junior 76 (ASE) \\
\hline Acrostichum aureum $\mathrm{L}$. & Erv & - & NE, EMA & Santos, LAS 81 (ASE) \\
\hline Pityrogramma calomelanos (L.) Link & Erv & - & NE & Costa 453 (ASE) \\
\hline Vittaria lineata (L.) Sm. & Erv & NR & $\mathrm{NE}$ & Santiago, A 1320 (ASE) \\
\hline Selaginellaceae & & & & \\
\hline Selaginella sp. & Erv & - & $\mathrm{NE}$ & Santos, LAS 87 (ASE) \\
\hline \multicolumn{5}{|c|}{ GIMNOSPERMAS } \\
\hline \multicolumn{5}{|l|}{ Podocarpaceae } \\
\hline Podocarpus sellowii Klotzsch ex Endl. & Arv & - & LC & Fonseca s/n (ASE 3) \\
\hline \multicolumn{5}{|c|}{ ANGIOSPERMAS } \\
\hline \multicolumn{5}{|l|}{ Acanthaceae } \\
\hline Ruellia asperula (Mart. ex Ness) Lindau & Arb & - & NE, ECA & Silva s/n (ASE 5376) \\
\hline Ruellia bahiensis (Nees) Morong & Arb & NR & NE, ECA & Gomes 22 (ASE) \\
\hline Ruellia cearensis Lindau & Arb & - & $\mathrm{NE}$ & Mendes 131 (ASE) \\
\hline Alstroemeriaceae & & & & \\
\hline $\begin{array}{l}\text { Alstroemeria longistaminea Mart. ex } \\
\text { Schult. \& Schult.f. }\end{array}$ & Erv & - & NE, ECA & Prata, AP 1356 (ASE) \\
\hline Bomarea edulis (Tussac) Herb. & Tre-H & - & $\mathrm{NE}$ & Nascimento-Junior 103 (ASE) \\
\hline
\end{tabular}


Tabela 1. Continuação.

\begin{tabular}{|c|c|c|c|c|}
\hline Família/Espécie & HAB & OCO & CAT & Coletor no./Autor(es) \\
\hline \multicolumn{5}{|l|}{ Amaranthaceae } \\
\hline Gomphrena demissa Mart. & Erv & - & $\mathrm{NE}$ & Viana 1972 (ASE) \\
\hline \multicolumn{5}{|l|}{ Amaryllidaceae } \\
\hline Griffinia espiritensis Ravenna & Erv & NR & RAR, EN, EMA & Barreto, ACG s/n (ASE 231) \\
\hline Hippeastrum stylosum Herb. & Erv & - & $\mathrm{NE}$ & Prata, AP 2910 (ASE) \\
\hline Zephyranthes cearensis (Herb.) Baker & Erv & - & NE & Landim, M 1413 (ASE) \\
\hline \multicolumn{5}{|l|}{ Anacardiaceae } \\
\hline Anacardium occidentale L. & Arv & - & NE & Matos, ECA 25 (ASE) \\
\hline Tapirira guianensis Aubl. & Arv & - & $\mathrm{NE}$ & Mendes, K 129 (ASE) \\
\hline Thyrsodium spruceanum Benth. & Arv & - & $\mathrm{NE}$ & Viana, G 877 (ASE) \\
\hline \multicolumn{5}{|l|}{ Annonaceae } \\
\hline Annona salzmannii A.DC. & Arv & - & NE, EMA & Costa, EV 16 (ASE) \\
\hline Duguetia gardneriana Mart. & Arv & - & $\mathrm{NE}$ & Gomes, LA 560 (ASE) \\
\hline Duguetia moricandiana Mart. & Arv & - & NE, EMA & Silva, FO 35 (ASE) \\
\hline Guatteria pogonopus Mart. & Arv & NR & $\mathrm{NE}$ & Moura, D 785 (ASE) \\
\hline Xylopia frutescens Aubl. & Arv & - & NE & Gomes, LA 562 (ASE) \\
\hline Xylopia laevigata (Mart.) R.E.Fr. & Arv & - & NE & Costa, EV 3 (ASE) \\
\hline \multicolumn{5}{|l|}{ Apocynaceae } \\
\hline Asclepias mellodora A.St.-Hil. & Erv & - & LC & Carregosa, T 12 (ASE) \\
\hline Blepharodon costae Fontella \& Morillo & Tre-H & - & NE, EMA & Carneiro, EM 382 (ASE) \\
\hline Blepharodon pictum (Vahl) W.D.Stevens & Tre-H & - & $\mathrm{NE}$ & Farinaccio, MA 866 (ASE) \\
\hline Cryptostegia madagascariensis Bojer & Arb & NR & $\mathrm{NE}$ & Silva, FO 147 (ASE) \\
\hline Ditassa crassifolia Decne. & Tre-H & - & $\mathrm{NE}$ & Nascimento-Junior 127 (ASE) \\
\hline Ditassa hispida (Vell.) Fontella & Tre-H & - & NE & Lucena, MFA 1804 (ASE) \\
\hline \multicolumn{5}{|l|}{ K.Schum. } \\
\hline Hancornia speciosa Gomes & Arv & - & $\mathrm{NE}$ & Iran s/n (ASE 10889) \\
\hline Himatanthus bracteatus (A.DC.) Woodson & Arv & - & $\mathrm{NE}$ & Matos, ECA 247 (ASE) \\
\hline $\begin{array}{l}\text { Himatanthus obovatus } \quad \text { (Müll.Arg.) } \\
\text { Woodson }\end{array}$ & \multicolumn{3}{|c|}{ Woodson } & Costa, SM 417 (ASE) \\
\hline Mandevilla hirsuta (A.Rich.) K.Schum. & Tre-L & - & LC & Viana, G 656 (ASE) \\
\hline $\begin{array}{l}\text { Mandevilla microphylla } \quad \text { (Stadelm.) } \\
\text { M.F.Sales \& Kin.-Gouv. }\end{array}$ & Tre-L & NR & $\mathrm{NE}$ & Ferreira, AC 6 (ASE) \\
\hline Mandevilla moricandiana (A.DC.) Woodson & Tre-L & - & NE & Farinaccio, MA 824 (ASE) \\
\hline $\begin{array}{l}\text { Mandevilla scabra (Hoffmanns. ex Roem. } \\
\text { \& Schult.) K.Schum. }\end{array}$ & Tre-L & - & $\mathrm{NE}$ & Farinaccio, MA 833 (ASE) \\
\hline Mandevilla tenuifolia (J.C.Mikan) Woodson & Tre-H & - & NE & Barreto, MI 27 (ASE) \\
\hline Matelea ganglinosa (Vell.) Rapini & Tre-H & - & $\mathrm{NE}$ & Nascimento-Junior 131 (ASE) \\
\hline Matelea orthosioides (E. Fourn.) Fontella & Tre-H & - & $\mathrm{NE}$ & Farinaccio, MA 827 (ASE) \\
\hline Tabernaemontana pandacaqui Poir. & Arb & NR & $\mathrm{NE}$ & Lima, JS 121 (ASE) \\
\hline Temnadenia odorifera (Vell.) J.F. Morales & Tre-L & - & NE, EMA & Costa, SM 307 (ASE) \\
\hline \multicolumn{5}{|l|}{ Aquifoliaceae } \\
\hline Ilex affinis Gardner & Arv & - & $\mathrm{NE}$ & Farinaccio, MA 759 (ASE) \\
\hline Ilex dumosa Reissek & Arv & - & NE & Araujo, D 1964 (ASE) \\
\hline \multicolumn{5}{|l|}{ Araceae } \\
\hline Anthurium affine Schott & Erv & - & $\mathrm{NE}$ & Matos, ECA 250 (ASE) \\
\hline Anthurium bellum Schott & Erv & - & NE, EMA & Nascimento-Junior 541 (ASE) \\
\hline Anthurium gracile (Rudge) Lindl. & Erv & - & $\mathrm{NE}$ & Mendes et al. (2010) \\
\hline Anthurium longipes N.E.Br. & Erv & - & NE, EMA & W Thomas 8893 (CEPEC) \\
\hline Dracontioides desciscens (Schott) Engl. & Erv & - & LC, EMA & Nascimento-Junior 324 (ASE) \\
\hline Philodendron acutatum Schott & Erv-Epi & - & $\mathrm{NE}$ & Oliveira, R 16 (ASE) \\
\hline $\begin{array}{l}\text { Philodendron fragrantissimum (Hook.) } \\
\text { G.Don }\end{array}$ & Erv-Epi & - & $\mathrm{NE}$ & Amorim, BS 294 (ASE) \\
\hline \multicolumn{5}{|l|}{ Araliaceae } \\
\hline Hydrocotyle sp. & Erv & - & NE & Viana, G 1116 (ASE) \\
\hline $\begin{array}{l}\text { Schefflera morototoni (Aubl.) Maguire, } \\
\text { Steyerm. \& Frodin }\end{array}$ & Arv & - & NE & Vicente, A 164 (ASE) \\
\hline \multicolumn{5}{|l|}{ Arecaceae } \\
\hline Allagoptera arenaria (Gomes) Kuntze & Pal & - & LC, EMA & Gomes, P 624 (ASE) \\
\hline Allagoptera brevicalyx M.Moraes & Pal & - & VU & Almeida, C 12 (ASE) \\
\hline Allagoptera campestris (Mart.) Kuntze & Pal & NR & $\mathrm{NE}$ & Fonseca, MR s/n (ASE 152) \\
\hline
\end{tabular}


Tabela 1. Continuação.

\begin{tabular}{|c|c|c|c|c|}
\hline Família/Espécie & HAB & OCO & CAT & Coletor no./Autor(es) \\
\hline Attalea funifera Mart. & $\mathrm{Pal}$ & - & NT, EMA & Gomes, P 813 (UFP) \\
\hline Bactris acanthocarpa Mart. & $\mathrm{Pal}$ & NR & NE & Mendes et al. (2010) \\
\hline Bactris horridispatha Noblick ex A.J. Hend. & Pal & NR & NE, EMA & Córdula, E 171 (UFP) \\
\hline Desmoncus sp. & Pal & - & $\mathrm{NE}$ & Mendes et al. (2010) \\
\hline Geonoma pohliana Mart. & $\mathrm{Pal}$ & NR & NE, EMA & Gomes, P 788 (ASE) \\
\hline Syagrus coronata (Mart.) Becc. & Pal & - & NE & Matos, ECA 151 (ASE) \\
\hline Syagrus schizophylla (Mart.) Glassman & Pal & - & NT, EMA & Santos, LAS 539 (ASE) \\
\hline \multicolumn{5}{|l|}{ Aristolochiaceae } \\
\hline Aristolochia birostris Duch & Tre-H & - & $\mathrm{NE}$ & Santos, ACAS 30 (ASE) \\
\hline Aristolochia labiata Willd. & Tre-H & - & LC & Dantas, TVP 64 (ASE) \\
\hline \multicolumn{5}{|l|}{ Asteraceae } \\
\hline Achyrocline satureioides (Lam.) DC. & Erv & NR & $\mathrm{NE}$ & Vicente, A 36 (ASE) \\
\hline Acmella uliginosa (Sw.) Cass. & Erv & NR & $\mathrm{NE}$ & Santos, ACAS 28 (ASE) \\
\hline $\begin{array}{l}\text { Acritopappus confertus } \quad \text { (Gardner) } \\
\text { R.M.King \& H.Rob. }\end{array}$ & \multicolumn{3}{|c|}{ R.M.King \& H.Rob. } & Souza, CL 12 (ASE) \\
\hline Ageratum conyzoides $\mathrm{L}$. & Erv & - & $\mathrm{NE}$ & Menezes, AB 77 (ASE) \\
\hline Albertinia brasiliensis Spreng. & Arb & - & $\mathrm{NE}$ & Vicente, A 10 (ASE) \\
\hline Aspilia foliacea (Spreng.) Baker & Erv & - & $\mathrm{NE}$ & Nascimento-Junior 154 (ASE) \\
\hline Aspilia itabaianensis J.U.Santos & Erv & - & NE, ECA & Fabricante, JR 106 (ASE) \\
\hline Aspilia martii Baker & Erv & - & NE & Dantas, TVP 25 (ASE) \\
\hline Centratherum punctatum Cass. & Erv & - & $\mathrm{NE}$ & Matos, ECA 4 (ASE) \\
\hline Chaptalia integerrima (Vell.) Burkart & Erv & NR & $\mathrm{NE}$ & Costa, SM 323 (ASE) \\
\hline $\begin{array}{l}\text { Conocliniopsis prasiifolia (DC.) R.M. King } \\
\& \text { H. Rob. }\end{array}$ & Erv & NR & $\mathrm{LC}$ & Nascimento-Junior 139 (ASE) \\
\hline Conyza bonariensis (L.) Cronquist & Erv & - & $\mathrm{NE}$ & Barreto, MI 28 (ASE) \\
\hline Cyrtocymura scorpioides (Lam.) H.Rob. & Arb & - & $\mathrm{NE}$ & Mendes et al. (2010) \\
\hline Delilia biflora (L.) Kuntze & Erv & - & $\mathrm{NE}$ & Santos, ACAS 65 (ASE) \\
\hline Eclipta prostrata L. & Erv & - & $\mathrm{NE}$ & Viana, G 536 (ASE) \\
\hline Elephantopus hirtiflorus DC. & Arb & - & $\mathrm{NE}$ & Dantas, TVP 79 (ASE) \\
\hline Emilia sonchifolia (L.) DC. Ex wight & Erv & - & $\mathrm{NE}$ & Dantas, TVP 61 (ASE) \\
\hline Erechtites hieracifolius (L.) Raf. ex DC & Erv & - & $\mathrm{NE}$ & Dantas, TVP 58 (ASE) \\
\hline Ichthyothere terminalis (Spreng.) S.F.Blake & Erv & - & $\mathrm{NE}$ & Silva, FO 56 (ASE) \\
\hline $\begin{array}{l}\text { Lepidaploa chalybaea (Mart. ex DC.) } \\
\text { H.Rob. }\end{array}$ & Sub & NR & $\mathrm{NE}$ & Machado, WJ 1226 (ASE) \\
\hline Lepidaploa mucronifolia (DC.) H.Rob. & Arb & - & $\mathrm{NE}$ & Moura, D 764 (ASE) \\
\hline Lessingianthus sp. & Arb & - & $\mathrm{NE}$ & Santos, ACAS 26 (ASE) \\
\hline Melanthera sp. & Erv & - & $\mathrm{NE}$ & Vivi, SV s/n (ASE 11588) \\
\hline Mikania cordifolia (L.f.) Willd. & Tre-L & - & $\mathrm{NE}$ & Matos, ECA 55 (ASE) \\
\hline Mikania nodulosa Sch. Bip. ex Baker & Tre-L & NR & NT, EMA & Santos, ML 22 (ASE) \\
\hline $\begin{array}{l}\text { Moquiniastrum oligocephalum (Gardner) } \\
\text { G. Sancho }\end{array}$ & Arb & NR & $\mathrm{NE}$ & Nascimento-Junior, JE 209 (ASE) \\
\hline $\begin{array}{l}\text { Paralychnophora reflexoauriculata (G.M. } \\
\text { Barroso) MacLeish }\end{array}$ & Arv & - & NT & Fonseca, MR s/n (ASE 6826) \\
\hline $\begin{array}{l}\text { Platypodanthera melissifolia } \quad \text { (DC.) } \\
\text { R.M.King \& H.Rob. }\end{array}$ & Erv & - & $\mathrm{NE}$ & Viana, G 96 (ASE) \\
\hline Porophyllum sp. & Erv & - & $\mathrm{NE}$ & Mendes et al. (2010) \\
\hline Sphagneticola trilobata (L.) Pruski & Erv & - & $\mathrm{NE}$ & Silva, FO 202 (ASE) \\
\hline Stenocephalum sp. & Erv & - & $\mathrm{NE}$ & Santos, ACAS 26 (ASE) \\
\hline Tilesia baccata (L.) Pruski & Arb & - & $\mathrm{NE}$ & Nascimento-Junior, JE 124 (ASE) \\
\hline Verbesina macrophylla (Cass.) S.F. Blake & Arb & - & $\mathrm{NE}$ & Matos, ECA 27 (ASE) \\
\hline Vernonanthura brasiliana (L.) H.Rob. & Arb & NR & $\mathrm{NE}$ & Vicente, A 34 (ASE) \\
\hline Vernonia sp. & Arb & - & $\mathrm{NE}$ & Nascimento-Junior, JE 92 (ASE) \\
\hline Wedelia sp. & Erv & - & $\mathrm{NE}$ & Gomes, E 13 (ASE) \\
\hline \multicolumn{5}{|l|}{ Begoniaceae } \\
\hline Begonia reniformis Dryand. & Erv & - & $\mathrm{NE}$ & Vicente, A 172 (ASE) \\
\hline Begonia saxicola A. DC. & Erv & NR & $\mathrm{NE}$ & Wellington 4 (ASE) \\
\hline \multicolumn{5}{|l|}{ Bignoniaceae } \\
\hline Adenocalymma coriaceum A.DC. & Tre-L & NR & $\mathrm{NE}$ & Silva, FO 199 (ASE) \\
\hline $\begin{array}{l}\text { Amphilophium } \quad \text { crucigerum } \\
\text { L.G.Lohmann }\end{array}$ & Tre-L & - & $\mathrm{NE}$ & Silva, FO 219 (ASE) \\
\hline Bignonia corymbosa (Vent.) L.G. Lohmann & Tre-L & - & $\mathrm{NE}$ & Saka, MN 212 (ASE) \\
\hline Jacaranda caroba (Vell.) DC. & Arv & NR & $\mathrm{NE}$ & Lucena, MFA 1355 (ASE) \\
\hline
\end{tabular}


Tabela 1. Continuação.

\begin{tabular}{|c|c|c|c|c|c|}
\hline Família/Espécie & $\overline{\mathrm{HAB}}$ & OCO & CAT & Coletor no./Autor(es) & \\
\hline Jacaranda jasminoides (Thumb.) Sandwith & Arv & - & $\mathrm{NE}$ & Vicente, A 21 (ASE) & \\
\hline Jacaranda obovata Cham. & Arv & - & NE & Santos, LAS 75 (ASE) & \\
\hline \multicolumn{6}{|l|}{ Bonnetiaceae } \\
\hline $\begin{array}{l}\text { Bonnetia stricta (Nees) Nees \& Mart. } \\
\text { Boraginaceae }\end{array}$ & \multicolumn{4}{|c|}{ Boraginaceae } & \\
\hline Cordia pilosa M. Stapf \& Taroda & Arb & - & NE, EMA & $\begin{array}{l}\text { Nascimento-Junior } \quad \mathrm{s} / \mathrm{n} \\
11290)\end{array}$ & (ASE \\
\hline Cordia superba Cham. & Arv & - & $\mathrm{NE}$ & Alves-Araújo, A 1110 (UFP) & \\
\hline Cordia toqueve Aubl. & Arv & - & $\mathrm{NE}$ & Vicente, A 160 (ASE) & \\
\hline $\begin{array}{l}\text { Myriopus villosus (Salzm. ex DC.) J.I.M. } \\
\text { Melo }\end{array}$ & Arb & - & $\mathrm{NE}$ & Matos, ECA 40 (ASE) & \\
\hline Varronia curassavica Jacq. & Arb & - & $\mathrm{NE}$ & Viana, G 1121 (ASE) & \\
\hline $\begin{array}{l}\text { Varronia johnstoniana J.I.M. Melo \& D.D. } \\
\text { Vieira }\end{array}$ & Sub & - & $\mathrm{NE}$ & Melo \& Vieira (2015) & \\
\hline Varronia multispicata (Cham.) Borhidi & Arb & - & NE & Costa, SM 333 (ASE) & \\
\hline Varronia polycephala Lam. & Arb & NR & $\mathrm{NE}$ & Prata, AP 2901 (ASE) & \\
\hline \multicolumn{6}{|l|}{ Bromeliaceae } \\
\hline Aechmea aquilega (Salisb.) Griseb. & Erv & - & $\mathrm{NE}$ & Matos, ECA 218 (ASE) & \\
\hline Aechmea bromeliifolia (Rudge) Baker & Erv & NR & LC & Santana, MC s/n (ASE 1114) & \\
\hline Aechmea costantinii (Mez) L.B.Sm. & Erv & NR & NE, EMA & Fonseca, MR s/n (ASE 160) & \\
\hline $\begin{array}{l}\text { Aechmea froesii ( } \text { L.B.Sm.) Leme \& } \\
\text { J.A.Siqueira }\end{array}$ & & Amorim, BS 300 (UFP) & \\
\hline Aechmea marauensis Leme & Erv & NR & NE, EMA & Buta, JM 5 (ASE) & \\
\hline $\begin{array}{l}\text { Aechmea mertensii (G. Mey) Schult. } \\
\text { \&Schult.f. }\end{array}$ & Erv-Epi & - & $\mathrm{NE}$ & Santos, LAS 84 (ASE) & \\
\hline Aechmea multiflora L.B. Sm & Erv & - & NE, EMA & Gomes, P 634 (ASE) & \\
\hline Aechmea nudicaulis (L.) Griseb. & Erv & - & LG & Landim, M 1393 (ASE) & \\
\hline $\begin{array}{l}\text { Aechmea patentissima (Mart. Ex Schult. \& } \\
\text { Schult.f.) Baker }\end{array}$ & Erv & - & NE, EMA & Amorim, BS 299 (UFP) & \\
\hline Cryptanthus sergipensis I. Ramírez & Erv & - & NE, EMA & Amorim, BS 304 (ASE) & \\
\hline Cryptanthus zonatus (Vis.) Bee & Erv & - & VU, EMA & Mendes, K 228 (UFP) & \\
\hline Guzmania lingulata (L.) Mez & Erv-Epi & - & NE & Mendes, K 225 (UFP) & \\
\hline Hohenbergia catingae Ule & Erv & - & $\mathrm{NE}$ & Prata, AP 1461 (ASE) & \\
\hline Hohenbergia horrida Harms & Erv & NR & NE, ECA & Landim, M 1406 (ASE) & \\
\hline $\begin{array}{l}\text { Racinaea spiculosa (Griseb.) M.A.Spencer \& } \\
\text { L.B.Sm. }\end{array}$ & Erv & - & LC & Santana, MC s/n (ASE 1115) & \\
\hline Tillandsia bulbosa Hook.f. & Erv-Epi & - & $\mathrm{NE}$ & Amorim, BS 298 (ASE) & \\
\hline Tillandsia gardneri Lindl. & Erv-Epi & - & LG & Melo, DS 58 (ASE) & \\
\hline Tillandsia polystachia (L.) L. & Erv-Epi & - & $\mathrm{NE}$ & Melo, DS 60 (ASE) & \\
\hline Tillandsia recurvata (L.) L. & Erv-Epi & - & $\mathrm{NE}$ & Melo, DS 59 (ASE) & \\
\hline Tillandsia stricta Sol. & Erv-Epi & - & $\mathrm{NE}$ & $\begin{array}{l}\text { Nascimento-Junior } 106 \\
11290 \text { ) }\end{array}$ & (ASE \\
\hline Tillandsia usneoides $(\mathrm{L}.) \mathrm{L}$. & Erv-Epi & - & LC & Carregosa, T 208 (ASE) & \\
\hline Vriesea scalaris E. Morren & Erv & NR & LC, EMA & Viana, G 103 (ASE) & \\
\hline Vriesea simplex (Vell.) Beer & Erv-Epi & NR & NE, EMA & Santos, JL 35 (ASE) & \\
\hline \multicolumn{6}{|l|}{ Burmanniaceae } \\
\hline Apteria aphylla (Nutt.) Barnhart ex Small & Erv-Sap & - & LC & Mendes, K 295 (ASE) & \\
\hline Burmannia capitata (Walter ex J.F. Gmel.) & Erv-Sap & - & $\mathrm{NE}$ & Gomes, P 605 (ASE) & \\
\hline \multicolumn{6}{|l|}{ Mart. } \\
\hline \multicolumn{6}{|l|}{ Burseraceae } \\
\hline Protium heptaphyllum (Aubl.) Marchand & Arv & - & NE & Mendes, K 350 (ASE) & \\
\hline Protium occhionii Rizzini & Arb & - & EN, EMA & Landim, M 890 (ASE) & \\
\hline \multicolumn{6}{|l|}{ Cactaceae } \\
\hline Epiphyllum phyllanthus (L.) Haw. & Arb-Epi & - & LC & Amorim, BS 305 (UFP) & \\
\hline $\begin{array}{l}\text { Melocactus violaceus subsp. margaritaceus } \\
\text { N.P.Taylor }\end{array}$ & Erv & - & NE, EMA & Gomes, P 633 (ASE) & \\
\hline $\begin{array}{l}\text { Pilosocereus catingicola } \\
\text { salvadorensis (Werderm.) Zappi }\end{array}$ & Arb & - & $\mathrm{NE}$ & Carregosa, T 58 (ASE) & \\
\hline $\begin{array}{l}\text { Rhipsalis floccosa subsp. floccosa Salm- } \\
\text { Dyck ex Pfeiff. }\end{array}$ & Arb-Epi & - & $\mathrm{NE}$ & Araujo, D 1965 (ASE) & \\
\hline $\begin{array}{l}\text { Calophyllaceae } \\
\text { Kielmeyera argentea Choisy }\end{array}$ & Arv & NR & NE, EMA & Santos, ML 105 (ASE) & \\
\hline
\end{tabular}


Tabela 1. Continuação.

\begin{tabular}{|c|c|c|c|c|}
\hline Família/Espécie & HAB & OCO & CAT & Coletor no./Autor(es) \\
\hline Kielmeyera petiolaris Mart. \& Zucc. & Arv & NR & $\mathrm{NE}$ & Gilvan s/n (ASE 13765) \\
\hline Kielmeyera rugosa Choisy & Arv & - & NE, EMA & Viana, G 1281 (ASE) \\
\hline \multicolumn{5}{|l|}{ Campanulaceae } \\
\hline Centropogon cornutus (L.) Druce & Arb & - & $\mathrm{NE}$ & Vicente, A 162 (ASE) \\
\hline \multicolumn{5}{|l|}{ Cannabaceae } \\
\hline Celtis iguanaea (Jacq.) Sarg. & Arv & - & $\mathrm{NE}$ & Andrade-Lima, D s/n (ASE 1238) \\
\hline Trema micrantha (L.) Blume & Arv & - & $\mathrm{NE}$ & Mendes, K 258 (ASE) \\
\hline \multicolumn{5}{|l|}{ Capparaceae } \\
\hline Cynophalla flexuosa (L.) J.Presl & Arb & - & $\mathrm{NE}$ & Fonseca, MR 862 (ASE) \\
\hline Dactylaena microphylla Eichler & Arb & - & $\mathrm{NE}$ & Andrade-Lima, D 62 (IPA) \\
\hline \multicolumn{5}{|l|}{ Celastraceae } \\
\hline Hippocratea volubilis $\mathrm{L}$. & Tre-L & - & $\mathrm{NE}$ & Mendes et al. (2010) \\
\hline Maytenus obtusifolia Mart. & Arv & - & $\mathrm{NE}$ & Santos, LAS 483 (ASE) \\
\hline \multicolumn{5}{|l|}{ Chrysobalanaceae } \\
\hline Couepia impressa Prance & Arv & NR & NE, EMA & Mendes et al. (2010) \\
\hline $\begin{array}{l}\text { Couepia uiti (Mart. \& Zucc.) Benth. ex } \\
\text { Hook f. }\end{array}$ & Arv & - & $\mathrm{NE}$ & Viana, G 1953 (ASE) \\
\hline Hirtella ciliata Mart. \& Zucc. & Arb & - & $\mathrm{NE}$ & $\begin{array}{l}\text { Nascimento-Junior } \quad \mathrm{s} / \mathrm{n} \quad \text { (ASE } \\
\text { 11285) }\end{array}$ \\
\hline Hirtella martiana Hook.f. & Arb & NR & $\mathrm{NE}$ & Mendes, K 290 (ASE) \\
\hline Hirtella racemosa Lam. & Arb & - & LC & Matos, ECA 221 (ASE) \\
\hline Licania sp. & Arv & - & $\mathrm{NE}$ & Landim, M 673 (ASE) \\
\hline \multicolumn{5}{|l|}{ Cleomaceae } \\
\hline Physostemon guianense (Aubl.) Malme & Arb & - & $\mathrm{NE}$ & Schimdt, S 219 (ASE) \\
\hline \multicolumn{5}{|l|}{ Clusiaceae } \\
\hline Clusia nemorosa G.Mey. & Arv & - & $\mathrm{NE}$ & Santana, MC 147 (ASE) \\
\hline Clusia sellowiana Schltdl. & Arv & - & NE, EMA & Santos, ACAS 71 (ASE) \\
\hline Symphonia globulifera L.f. & Arv & - & $\mathrm{NE}$ & Vicente, A 191 (ASE) \\
\hline \multicolumn{5}{|l|}{ Commelinaceae } \\
\hline Commelina diffusa Burm.f. & Erv & - & $\mathrm{NE}$ & Araujo, DA 2234 (ASE) \\
\hline Commelina erecta $\mathrm{L}$. & Erv & - & $\mathrm{NE}$ & Matos, ECA 229 (ASE) \\
\hline Commelina obliqua Vahl & Erv & - & $\mathrm{NE}$ & Mendes et al. (2010) \\
\hline Dichorisandra hexandra (Aubl.) Standl. & Tre-H & - & $\mathrm{NE}$ & Gomes, P 771 (ASE) \\
\hline Dichorisandra procera Mart. ex Schult. f. & Erv & - & NE, EMA & Viana, G 1268 (ASE) \\
\hline \multicolumn{5}{|l|}{ Connaraceae } \\
\hline Connarus sp. & Arv & - & $\mathrm{NE}$ & Fonseca, MR s/n (ASE 424) \\
\hline \multicolumn{5}{|l|}{ Convolvulaceae } \\
\hline $\begin{array}{l}\text { Daustinia montana (Moric.) Buril \& A.R. } \\
\text { Simões }\end{array}$ & Tre-L & - & $\mathrm{NE}$ & Viana, G 1240 (ASE) \\
\hline Evolvulus echioides Moric. & Arb & NR & NE, ECA & Lucena, MFA 1764 (ASE) \\
\hline Evolvulus fuscus Meisn. & Arb & NR & NT & Amorim, BS 149 (ASE) \\
\hline Evolvulus glomeratus Nees \& C. Mart. & Arb & - & $\mathrm{NE}$ & Fonseca, MR 877 (ASE) \\
\hline Evolvulus imbricatus Mart. ex Colla & Arb & - & NE, EMA & Viana, G 1314 (ASE) \\
\hline Evolvulus lithospermoides Mart. & Arb & NR & $\mathrm{NE}$ & Costa, SM 429 (ASE) \\
\hline Evolvulus phyllanthoides Moric. & Arb & - & $\mathrm{NE}$ & Nascimento-Junior 102 (ASE) \\
\hline Evolvulus pterocaulon Moric. & Arb & - & $\mathrm{NE}$ & Carneiro, EM 350 (ASE) \\
\hline Evolvulus sericeus $\mathrm{Sw}$. & Arb & - & $\mathrm{NE}$ & Nascimento-Junior 195 (ASE) \\
\hline Evolvulus thymiflorus Choisy & Arb & - & $\mathrm{NE}$ & Viana, G 1273 (ASE) \\
\hline Ipomoea amnicola Morong & Tre-L & NR & $\mathrm{NE}$ & Mendes et al. (2010) \\
\hline $\begin{array}{l}\text { Ipomoea bahiensis Willd. ex Roem. \& } \\
\text { Schult. }\end{array}$ & Tre-L & - & $\mathrm{NE}$ & Mendes et al. (2010) \\
\hline Ipomoea incarnata (Vahl.) Choiby & Tre-L & - & $\mathrm{NE}$ & Mendes et al. (2010) \\
\hline Ipomoea quamoclit $\mathrm{L}$. & Tre-L & - & $\mathrm{NE}$ & Mendes, K 257 (HUEFS) \\
\hline Jacquemontia bahiensis O'Donell & Tre-L & - & $\mathrm{NE}$ & Viana, G 1973 (ASE) \\
\hline Jacquemontia blanchetii Moric. & Tre-L & - & $\mathrm{NE}$ & Matos, GMA 166 (ASE) \\
\hline Jacquemontia bracteosa Meisn. & Tre-L & - & $\mathrm{NE}$ & Santos, ACAS 7 (ASE) \\
\hline Jacquemontia evolvuloides (Moric.) Meisn. & Tre-L & - & $\mathrm{NE}$ & Prata, AP 2782 (ASE) \\
\hline Jacquemontia nodiflora (Desr.) G.Don & Tre-L & - & $\mathrm{NE}$ & Amorim, BS 39 (ASE) \\
\hline Jacquemontia pentanthos (Jacq.) G. Don. & Tre-L & - & $\mathrm{NE}$ & Amorim, BS 173 (ASE) \\
\hline Jacquemontia velutina Choisy & Tre-L & NR & $\mathrm{NE}$ & Viana, G 1808 (ASE) \\
\hline Operculina macrocarpa (L.) Urb. & Tre-L & - & LG & Mendes et al. (2010) \\
\hline
\end{tabular}


Tabela 1. Continuação.

\begin{tabular}{|c|c|c|c|c|}
\hline Família/Espécie & HAB & OCO & CAT & Coletor no./Autor(es) \\
\hline \multicolumn{5}{|l|}{ Costaceae } \\
\hline Costus spiralis (Jacq.) Roscoe & Erv & - & $\mathrm{NE}$ & Landim, M 1047 (ASE) \\
\hline \multicolumn{5}{|l|}{ Gucurbitaceae } \\
\hline Cayaponia tayuya (Vell.) Cogn. & Tre-L & NR & $\mathrm{NE}$ & Amorim, BS 201 (ASE) \\
\hline Cyclanthera pedata (L.) Schrad. & Tre-H & NR & $\mathrm{NE}$ & Araujo, DA 2241 (ASE) \\
\hline Gurania sp. & Tre-L & - & $\mathrm{NE}$ & Prata $2893(\mathrm{ASE})$ \\
\hline Luffa cylindrica (L.) M.Roem. & Tre-L & NR & NE & Paula 3639 (ASE) \\
\hline \multicolumn{5}{|l|}{ Cyclanthaceae } \\
\hline Sp. & Arb & - & $\mathrm{NE}$ & Viana, G 1135 (ASE) \\
\hline \multicolumn{5}{|l|}{ Cyperaceae } \\
\hline Abildgaardia baeothryon A.St.-Hil. & Erv & - & $\mathrm{NE}$ & Santos 85 (ASE) \\
\hline Becquerelia cymosa Brongn. & Erv & - & $\mathrm{NE}$ & Menezes 67 (ASE) \\
\hline Bulbostylis capillaris (L.) C.B.Clarke & Erv & - & $\mathrm{NE}$ & Costa 401 (ASE) \\
\hline Bulbostylis conifera (Kunth) C.B.Clarke & Erv & - & $\mathrm{NE}$ & Nascimento-Junior 202 (ASE) \\
\hline Bulbostylis junciformis (Kunth) C.B.Clarke & Erv & - & $\mathrm{NE}$ & Nascimento-Junior 208 (ASE) \\
\hline $\begin{array}{l}\text { Bulbostylis scabra (J.Presl \& } \quad \text { C.Presl) } \\
\text { C.B.Clarke }\end{array}$ & Erv & - & $\mathrm{NE}$ & Gomes 595 (ASE) \\
\hline Bulbostylis tenuifolia (Rudge) J.F.Macbr. & Erv & - & $\mathrm{NE}$ & Maciel 312 (ASE) \\
\hline Cyperus aggregatus (Willd.) Endl. & Erv & - & $\mathrm{NE}$ & Nascimento-Junior 222 (ASE) \\
\hline Cyperus compressus $\mathrm{L}$. & Erv & - & $\mathrm{NE}$ & Costa 487 (ASE) \\
\hline Cyperus cuspidatus Kunth & Erv & - & $\mathrm{NE}$ & Costa 490 (ASE) \\
\hline Cyperus haspan $\mathrm{L}$ & Erv & - & $\mathrm{NE}$ & Costa 174 (ASE) \\
\hline Cyperus hermaphroditus (Jacq.) Standl. & Erv & - & $\mathrm{NE}$ & Costa 381 (ASE) \\
\hline Cyperus laxus Lam. & Erv & - & $\mathrm{NE}$ & Costa 461 (ASE) \\
\hline Cyperus ligularis L. & Erv & - & NE & Matos 248 (ASE) \\
\hline Cyperus luzulae (L.) Retz. & Erv & - & $\mathrm{NE}$ & Costa 415 (ASE) \\
\hline Cyperus meyenianus Kunth & Erv & - & $\mathrm{NE}$ & Maciel 296 (ASE) \\
\hline Cyperus odoratus $\mathrm{L}$. & Erv & - & $\mathrm{NE}$ & Silva 253 (ASE) \\
\hline Cyperus squarrosus L. & Erv & - & $\mathrm{NE}$ & Costa 489 (ASE) \\
\hline Cyperus surinamensis Rottb. & Erv & - & $\mathrm{NE}$ & Costa 177 (ASE) \\
\hline Eleocharis filiculmis Kunth & Erv & - & $\mathrm{NE}$ & Costa 176 (ASE) \\
\hline $\begin{array}{l}\text { Eleocharis maculosa (Vahl) Roem. \& } \\
\text { Schult. }\end{array}$ & Erv & - & $\mathrm{NE}$ & Costa 185 (ASE) \\
\hline Eleocharis nigrescens (Nees) Kunth & Erv & - & $\mathrm{NE}$ & Costa 437 (ASE) \\
\hline Fimbristylis complanata (Retz.) Link. & Erv & - & NE & Costa 181 (ASE) \\
\hline Fimbristylis cymosa $\mathrm{R} . \mathrm{Br}$. & Erv & - & NE & Costa 494 (ASE) \\
\hline Fimbristylis dichotoma (L.) Vahl & Erv & - & $\mathrm{NE}$ & Costa 501 (ASE) \\
\hline Fuirena umbellata Rottb. & Erv & - & NE & Costa 175 (ASE) \\
\hline Kyllinga brevifolia Rottb. & Erv & - & $\mathrm{NE}$ & Prata 2779 (ASE) \\
\hline Kyllinga odorata Vahl & Erv & - & $\mathrm{NE}$ & Costa 486 (ASE) \\
\hline Kyllinga squamulata Thonn. ex Vahl & Erv & - & $\mathrm{NE}$ & Costa 496 (ASE) \\
\hline Kyllinga vaginata Lam. & Erv & - & NE & Costa 178 (ASE) \\
\hline Lagenocarpus guianensis Lindl. ex Nees & Erv & - & $\mathrm{NE}$ & Menezes 60 (ASE) \\
\hline Lagenocarpus rigidus Nees & Erv & - & $\mathrm{NE}$ & Costa 445 (ASE) \\
\hline Lipocarpha micrantha (Vahl) G.C.Tucker & Erv & - & $\mathrm{NE}$ & Costa 485 (ASE) \\
\hline Lipocarpha salzmanniana Steud. & Erv & - & $\mathrm{NE}$ & Costa 444 (ASE) \\
\hline Pycreus fugax (Liebm.) C.D.Adams. & Erv & - & $\mathrm{NE}$ & Costa 488 (ASE) \\
\hline Pycreus polystachyos (Rottb.) P.Beauv. & Erv & - & NE & Costa 179 (ASE) \\
\hline Rhynchospora albiceps Kunth & Erv & - & $\mathrm{NE}$ & Costa 398 (ASE) \\
\hline Rhynchospora barbata (Vahl) Kunth & Erv & - & $\mathrm{NE}$ & Costa 393 (ASE) \\
\hline Rhynchospora cephalotes (L.) Vahl & Erv & - & $\mathrm{NE}$ & Matos 226 (ASE) \\
\hline Rhynchospora ciliata (Vahl) Kük. & Erv & - & $\mathrm{NE}$ & Lucena 1789 (ASE) \\
\hline Rhynchospora diodon (Nees) Griseb. & Erv & - & $\mathrm{NE}$ & Fonseca 879 (ASE) \\
\hline Rhynchospora emaciata (Nees) Boeckeler & Erv & - & $\mathrm{NE}$ & Lucena, MFA 1564 (UFP) \\
\hline Rhynchospora exaltata Kunth & Erv & - & NE & Maciel 311(ASE) \\
\hline Rhynchospora filiformis Vahl & Erv & - & LC & Costa 184 (ASE) \\
\hline $\begin{array}{l}\text { Rhynchospora holoschoenoides (Rich.) } \\
\text { Herter }\end{array}$ & Erv & - & $\mathrm{NE}$ & Costa 180 (ASE) \\
\hline Rhynchospora marisculus Lindl. ex Nees & Erv & - & NE & Santos 89 (ASE) \\
\hline Rhynchospora ridleyi C.B.Clarke & Erv & - & $\mathrm{NE}$ & Mendes 321 (ASE) \\
\hline Rhynchospora riedeliana C.B.Clarke & Erv & - & NE & Viana 1310 (ASE) \\
\hline Rhynchospora riparia (Nees) Boeckeler & Erv & - & $\mathrm{NE}$ & Costa 395(ASE) \\
\hline
\end{tabular}


Tabela 1. Continuação.

\begin{tabular}{|c|c|c|c|c|}
\hline Família/Espécie & HAB & $\mathrm{OCO}$ & CAT & Coletor no./Autor(es) \\
\hline Rhynchospora rugosa (Vahl) Gale & Erv & - & $\mathrm{NE}$ & Costa 186 (ASE) \\
\hline Rhynchospora tenuis Link & Erv & - & $\mathrm{NE}$ & Costa 409 (ASE) \\
\hline Scleria bracteata Cav. & Erv & - & $\mathrm{NE}$ & Matos 111 (ASE) \\
\hline Scleria cyperina Willd. ex Kunth & Erv & - & $\mathrm{NE}$ & Costa 193 (ASE) \\
\hline Scleria eggersiana Boeckeler & Erv & - & $\mathrm{NE}$ & Menezes 58 (ASE) \\
\hline Scleria interrupta Rich. & Erv & - & $\mathrm{NE}$ & Costa 404 (ASE) \\
\hline Scleria latifolia Sw. & Erv & - & $\mathrm{NE}$ & Menezes 61 (ASE) \\
\hline Scleria leptostachya Kunth & Erv & - & $\mathrm{NE}$ & Lucena 1759 (ASE) \\
\hline Scleria secans $(\mathrm{L}$.$) Urb.$ & Erv & - & $\mathrm{NE}$ & Menezes 63 (ASE) \\
\hline Scleria verticillata Muhl. Ex Willd. & Erv & - & $\mathrm{NE}$ & Costa 187 (ASE) \\
\hline \multicolumn{5}{|l|}{ Dilleniaceae } \\
\hline Curatella americana L. & Arv & - & $\mathrm{NE}$ & Nascimento-Junior 262 (ASE) \\
\hline Davilla flexuosa A.St.-Hil. & Arb & - & $\mathrm{NE}$ & Matos 234 (ASE) \\
\hline Davilla nitida (Vahl) Kubitzki & Arv & - & LC & Nascimento-Junior 263 (ASE) \\
\hline Tetracera boomii Aymard & Tre-L & - & NE, EMA & Mendes 227 (ASE) \\
\hline \multicolumn{5}{|l|}{ Dioscoreaceae } \\
\hline Dioscorea leptostachya Gardner & Tre-H & - & $\mathrm{NE}$ & Santos 574 (ASE) \\
\hline Dioscorea ovata Vell. & Tre-H & - & $\mathrm{NE}$ & Landim 817 (ASE) \\
\hline $\begin{array}{l}\text { Dioscorea piperifolia Humb \& Bonpl. ex } \\
\text { Willd. }\end{array}$ & Tre-H & - & $\mathrm{NE}$ & Matos 197 (ASE) \\
\hline \multicolumn{5}{|l|}{ Droseraceae } \\
\hline Drosera montana A.St.-Hil. & Erv & NR & $\mathrm{NE}$ & Amorim 144 (ASE) \\
\hline \multicolumn{5}{|l|}{ Elaeocarpaceae } \\
\hline $\begin{array}{l}\text { Sloanea guianensis (Aubl.) Benth. } \\
\text { Ericaceae }\end{array}$ & Arv & - & $\mathrm{NE}$ & Santos 26 (ASE) \\
\hline $\begin{array}{l}\text { Agarista revoluta (Spreng.) J.D. Hook. ex } \\
\text { Nied. }\end{array}$ & Arb & - & NE, EMA & Viana 1950 (ASE) \\
\hline \multicolumn{4}{|l|}{ Eriocaulaceae } & Fonseca s/n (ASE 100) \\
\hline Actinocephalus polyanthus (Bong.) Sano & Erv & NR & $\mathrm{NE}$ & Prata 2787 (ASE) \\
\hline Actinocephalus ramosus (Wikstr.) Sano & Erv & NR & $\mathrm{NE}$ & Carregosa 16 (ASE) \\
\hline $\begin{array}{l}\text { Comanthera imbricata (Körn.) L.R.Parra \& } \\
\text { Giul }\end{array}$ & Erv & - & $\mathrm{NE}$ & Fonseca 441 (ASE) \\
\hline Leiothrix flavescens (Bong.) Ruhland & Erv & - & $\mathrm{NE}$ & Mendes 81 (ASE) \\
\hline Leiothrix pilulifera (Körn.) Ruhland & Erv & NR & NE, EMA & Nascimento-Junior 90 (ASE) \\
\hline Paepalanthus bifidus (Schrad.) Kunth & Erv & - & NE & Prata 2781 (ASE) \\
\hline Paepalanthus myocephalus (Mart.) Körn. & Erv & - & $\mathrm{NE}$ & Nascimento-Júnior 112 (ASE) \\
\hline Paepalanthus pulchellus Herzog & Erv & NR & $\mathrm{NE}$ & Fonseca 511 (ASE) \\
\hline Paepalanthus tortilis (Bong.) Mart. & Erv & - & $\mathrm{NE}$ & Costa $172(\mathrm{ASE})$ \\
\hline Syngonanthus gracilis (Bong.) Ruhland & Erv & NR & $\mathrm{NE}$ & Matos 225 (ASE) \\
\hline Syngonanthus nitens Ruhland & Erv & - & $\mathrm{NE}$ & Fonseca, MR s/n (ASE 157) \\
\hline \multicolumn{5}{|l|}{ Erythroxylaceae } \\
\hline Erythroxylum citrifolium A.St.- Hil. & Arb & - & $\mathrm{NE}$ & Viana 1723 (ASE) \\
\hline Erythroxylum mucronatum Benth. & Arb & - & NE & Landim 300 (ASE) \\
\hline Erythroxylum nobile O.E.Schulz & Arb & - & NE, EMA & Santos 11 (ASE) \\
\hline Erythroxylum rimosum O.E.Schulg & Arb & - & $\mathrm{NE}$ & Vicente 110 (ASE) \\
\hline \multicolumn{5}{|l|}{ Euphorbiaceae } \\
\hline Aparisthmium cordatum (A. Juss) Baill. & Arv & - & $\mathrm{NE}$ & Santana, MC 137 (SPF) \\
\hline Astraea macroura (Colla) P.L.R. Moraes, De & Arb & - & $\mathrm{NE}$ & Viana, G 1739 (ASE) \\
\hline \multicolumn{5}{|l|}{ Smedt \& Guglielmone } \\
\hline Croton glandulosus $\mathrm{L}$. & Arb & - & $\mathrm{NE}$ & Lucena, MFA 1552 (UFP) \\
\hline Croton grewioides Baill. & Arb & - & NE, ECA & Santos 477 (ASE) \\
\hline Croton heliotropiifolius Kunth & Arb & - & NE & Santos 479 (ASE) \\
\hline Croton hirtus L'Hér. & Arb & - & LG & Amorim, BS s/n (UFP 47540) \\
\hline Croton lundianus (Didr.) Mül.Arg. & Arb & - & $\mathrm{NE}$ & Santos 31 (ASE) \\
\hline Croton pedicellatus Kunth & Arb & - & $\mathrm{NE}$ & Déda 66 (ASE) \\
\hline Croton sellowii Baill. & Arb & - & $\mathrm{NE}$ & Viana 875 (ASE) \\
\hline Dalechampia convolvuloides Lam. & Tre-H & - & $\mathrm{NE}$ & Lucena, MFA 1812 (UFP) \\
\hline Euphorbia comosa Vell. & Erv & - & $\mathrm{NE}$ & Costa 465 (ASE) \\
\hline Euphorbia heterophylla L. & Erv & - & $\mathrm{NE}$ & Nascimento-Junior 643(ASE) \\
\hline Euphorbia hirta L. & Erv & - & $\mathrm{NE}$ & Amorim, BS 313 (UFP) \\
\hline Euphorbia hyssopifolia L. & Erv & - & $\mathrm{NE}$ & Menezes 69 (ASE) \\
\hline
\end{tabular}


Tabela 1. Continuação.

\begin{tabular}{|c|c|c|c|c|}
\hline Família/Espécie & HAB & OCO & CAT & Coletor no./Autor(es) \\
\hline Euphorbia prostrata Aiton & Erv & - & $\mathrm{NE}$ & Amorim, BS 106 (UFP) \\
\hline Euphorbia thymifolia L. & Erv & - & $\mathrm{NE}$ & Matos $16(\mathrm{ASE})$ \\
\hline Microstachys corniculata (Vahl) Griseb. & Arb & - & $\mathrm{NE}$ & Matos 158 (ASE) \\
\hline Microstachys hispida (Mart.) Govaerts & Arb & NR & $\mathrm{NE}$ & Amorim, BS 311 (UFP) \\
\hline Sapium glandulosum (L.) Morong & Arv & - & $\mathrm{NE}$ & Lucena, MFA 1792 (UFP) \\
\hline Fabaceae & & & & \\
\hline Abarema filamentosa (Benth.) Pittier & Arv & - & LC, EMA & Alves-Araújo 961 (ASE) \\
\hline $\begin{array}{l}\text { Aeschynomene histrix var. incana (Vogel) } \\
\text { Benth. }\end{array}$ & Erv & NR & $\mathrm{NE}$ & Amorim 147 (ASE) \\
\hline Aeschynomene mollicula Kunth & Erv & NR & $\mathrm{NE}$ & Prata 2879 (ASE) \\
\hline Aeschynomene paniculata Willd. \& Vogel & Erv & - & $\mathrm{NE}$ & Oliveira 4 (ASE) \\
\hline Andira humilis Mart. ex Benth. & Arv & NR & NE & Barreto s/n (ASE 382) \\
\hline $\begin{array}{l}\text { Andira surinamensis (Bondt) Splitg. ex } \\
\text { Amshoff }\end{array}$ & Arv & NR & $\mathrm{NE}$ & Fonseca 513(ASE) \\
\hline Bauhinia acuruana Moric. & Arv & NR & $\mathrm{NE}$ & Dantas 36 (ASE) \\
\hline Bauhinia corifolia L.P.Queiroz & Arb & - & $\mathrm{NE}$ & Gomes 643 (ASE) \\
\hline Bowdichia virgilioides Kunth & Arv & - & NT & Andrade 24 (ASE) \\
\hline Camptosema sp. & Tre-L & - & $\mathrm{NE}$ & Santos, ACAS 20 (ASE) \\
\hline Centrosema brasilianum (L.) Benth. & Tre-H & - & $\mathrm{NE}$ & Dantas $62(\mathrm{ASE})$ \\
\hline Centrosema pascuorum Mart. ex Benth. & Tre-H & - & $\mathrm{NE}$ & Mendes et al. (2010) \\
\hline Centrosema virginianum (L.) Benth. & Tre-H & - & $\mathrm{NE}$ & Santos 113 (ASE) \\
\hline $\begin{array}{l}\text { Chamaecrista acosmifolia (Mart. ex Benth.) } \\
\text { H.S.Irwin e Barneby }\end{array}$ & Arb & NR & $\mathrm{NE}$ & Lucena, MFA 1772 (UFP) \\
\hline $\begin{array}{l}\text { Chamaecrista brachystachya (Benth.) Conc. } \\
\text { et al. }\end{array}$ & Arv & - & $\mathrm{NE}$ & Landim 524 (ASE) \\
\hline $\begin{array}{l}\text { Chamaecrista cytisoides (DC. ex Collad.) } \\
\text { H.S. Irwin \& Barneby }\end{array}$ & Arb & NR & RAR & Dantas 3 (ASE) \\
\hline $\begin{array}{l}\text { Chamaecrista desvauxii var. desvauxii } \\
\text { (Collad.) Killip }\end{array}$ & Erv & - & $\mathrm{NE}$ & Gomes 229 (ASE) \\
\hline $\begin{array}{l}\text { Chamaecrista desvauxii var. latistipula } \\
\text { (Benth.) G.P.Lewis }\end{array}$ & Arb & - & $\mathrm{NE}$ & Wellington 7 (ASE) \\
\hline $\begin{array}{l}\text { Chamaecrista ensiformis var. ensiformis } \\
\text { (Vell.) H.S.Irwin \& Barneby }\end{array}$ & Erv & - & $\mathrm{NE}$ & Viana 248 (ASE) \\
\hline Chamaecrista flexuosa (L.) Greene & Arv & - & $\mathrm{NE}$ & Carneiro 190 (ASE) \\
\hline $\begin{array}{l}\text { Chamaecrista hispidula (Vahl) H.S.Irwin \& } \\
\text { Barneby }\end{array}$ & Arb & - & $\mathrm{NE}$ & Gomes 594 (ASE) \\
\hline $\begin{array}{l}\text { Chamaecrista nictitans subsp. Patellaria } \\
\text { (DC. ex Collad.) H.S.Irwin \& Barneb }\end{array}$ & Erv & - & $\mathrm{NE}$ & Almeida s/n (ASE 11595) \\
\hline $\begin{array}{l}\text { Chamaecrista ramosa (Vogel) H.S. Irwin \& } \\
\text { Barneby }\end{array}$ & Arb & - & $\mathrm{NE}$ & Nascimento-Junior 121 (ASE) \\
\hline Chamaecrista rotundifolia (Pers.) Greene & Erv & - & $\mathrm{NE}$ & Viana 534 (ASE) \\
\hline $\begin{array}{l}\text { Chamaecrista swainsonii (Benth.) H.S.Irwin } \\
\text { \& Barneby }\end{array}$ & Arb & - & $\mathrm{NE}$ & Nascimento-Junior 170 (ASE) \\
\hline Chamaecrista unijuga (Benth.) Conc. et al. & Arv & - & NE, EMA & Carneiro, EM 113 (ASE) \\
\hline Clitoria guianensis (Aubl.) Benth. & Arv & NR & $\mathrm{NE}$ & Cynara s/n (ASE 10863) \\
\hline Clitoria laurifolia Poir. & Arv & - & LC & Marcos Antonio s/n (ASE 10854) \\
\hline Collaea speciosa (Loisel.) DC. & Arb & NR & LC & Mendes 89 (ASE) \\
\hline Cratylia mollis Mart. ex Benth. & Tre-L & NR & NE, ECA & Prata $2872(\mathrm{ASE})$ \\
\hline Crotalaria bahiensis Windler \& S.G.Skinner & Arb & NR & $\mathrm{NE}$ & Amorim, BS 192 (UFP) \\
\hline Crotalaria incana $\mathrm{L}$ & Arb & NR & $\mathrm{NE}$ & Luciano 6 (ASE) \\
\hline Crotalaria pallida Aiton & Arb & NR & $\mathrm{NE}$ & Matos 164 (ASE) \\
\hline Crotalaria pilosa Mill. & Arb & NR & $\mathrm{NE}$ & Viana 1262 (ASE) \\
\hline Dalbergia sp. & Arv & - & $\mathrm{NE}$ & Landim 1410 (ASE) \\
\hline Desmodium affine Schltdl. & Erv & - & $\mathrm{NE}$ & Prata 2892 (ASE) \\
\hline Desmodium barbatum (L.) Benth. & Arb & - & NE & Dantas $12(\mathrm{ASE})$ \\
\hline Desmodium tortuosum (Sw.) DC. & Erv & - & $\mathrm{NE}$ & Borba s/n (ASE 15607) \\
\hline Dioclea grandiflora Mart. ex Benth. & Tre-L & - & $\mathrm{NE}$ & Santos 114 (ASE) \\
\hline Dioclea lasiophylla Mart. ex Benth. & Tre-L & - & $\mathrm{NE}$ & Amorim 139 (ASE) \\
\hline Dioclea violacea Mart. ex Benth. & Tre-L & - & NE & Mendes et al. (2010) \\
\hline Erythrina velutina Willd. & Arv & - & $\mathrm{NE}$ & Viana 242 (ASE) \\
\hline Galactia sp. & Tre-H & - & NE & Viana $832(\mathrm{ASE})$ \\
\hline Hymenolobium alagoanum Ducke & Arv & - & NE, EMA & Nascimento-Junior 159 (ASE) \\
\hline
\end{tabular}


Tabela 1. Continuação.

\begin{tabular}{|c|c|c|c|c|}
\hline Família/Espécie & HAB & OCO & CAT & Coletor no./Autor(es) \\
\hline Inga laurina (Sw.) Willd & Arv & NR & LC & Carneiro 471 (ASE) \\
\hline Inga marginata Willd. & Arv & NR & $\mathrm{NE}$ & Vicente 42 (ASE) \\
\hline Leptolobium bijugum (Spreng.) Vogel & Arv & - & NE, EMA & Landim 506 (ASE) \\
\hline Libidibia ferrea (Mart. ex Tul.) L.P.Queiroz & Arv & - & $\mathrm{NE}$ & Vicente, A 163 (ASE) \\
\hline Machaerium hirtum (Vell.) Stellfeld & Arv & - & $\mathrm{NE}$ & Prata, AP 2887 (ASE) \\
\hline Mimosa pudica L. & Arb & NR & $\mathrm{NE}$ & Prata 2871 (ASE) \\
\hline $\begin{array}{l}\text { Mimosa somnians Humb. \& Bonpl. ex } \\
\text { Willd. }\end{array}$ & Arb & - & $\mathrm{NE}$ & Mendes 101 (ASE) \\
\hline Mimosa tenuiflora (Willd.) Poir. & Arb & - & $\mathrm{NE}$ & Mendes $163(\mathrm{ASE})$ \\
\hline Periandra mediterranea (Vell.) Taub. & Erv & - & $\mathrm{NE}$ & Nascimento-Junior 113 (ASE) \\
\hline Piptadenia stipulacea (Benth.) Ducke & Arb & - & $\mathrm{NE}$ & Fonseca $855(\mathrm{ASE})$ \\
\hline Rhynchosia phaseoloides (Sw.) DC. & Tre-H & NR & $\mathrm{NE}$ & Matos 37 (ASE) \\
\hline Tachigalisp. & Arv & - & $\mathrm{NE}$ & Landim, M 1289 (ASE) \\
\hline $\begin{array}{l}\text { Senna macranthera (DC. ex Collad.) } \\
\text { H.S.Irwin \& Barneby }\end{array}$ & Arv & NR & $\mathrm{NE}$ & Santana 71 (ASE) \\
\hline Senna obtusifolia (L.) H.S. Irwin \& Barneby & Arb & NR & $\mathrm{NE}$ & Santos 69 (ASE) \\
\hline $\begin{array}{l}\text { Senna pendula (Humb. \& Bonpl. ex Willd.) } \\
\text { H.S. Irwin \& Barneby }\end{array}$ & Arb & NR & $\mathrm{NE}$ & Matos 115 (ASE) \\
\hline Senna pinheiroi H.S.Irwin \& Barneby & Arb & - & $\mathrm{NE}$ & Alves-Araújo 943 (ASE) \\
\hline $\begin{array}{l}\text { Senna splendida (Vogel) H.S. Irwin \& } \\
\text { Barneby }\end{array}$ & Arb & - & $\mathrm{NE}$ & Santos 68 (ASE) \\
\hline $\begin{array}{l}\text { Stryphnodendron pulcherrimum (Willd.) } \\
\text { Hochr. }\end{array}$ & Arv & - & $\mathrm{NE}$ & Vicente 73 (ASE) \\
\hline Stylosanthes angustifolia Vogel & Erv & - & $\mathrm{NE}$ & Córdula, E. 146 (UFP) \\
\hline Stylosanthes capitata Vogel & Arb & - & $\mathrm{NE}$ & Amorim 156 (ASE) \\
\hline Stylosanthes gracilis Kunth & Arb & - & $\mathrm{NE}$ & Dantas, TVP 83 (ASE) \\
\hline Stylosanthes humilis Kunth & Erv & - & $\mathrm{NE}$ & Almeida, ES 351 (ASE) \\
\hline Stylosanthes scabra Vogel & Arb & NR & $\mathrm{NE}$ & Viana 1258 (ASE) \\
\hline Stylosanthes viscosa (L.) Sw. & Arb & - & $\mathrm{NE}$ & Dantas 86 (ASE) \\
\hline Swartzia apetala Raddi & Arv & - & $\mathrm{NE}$ & Schmidt 275 (ASE) \\
\hline Sweetia sp. & Erv & - & $\mathrm{NE}$ & Neto, JF 1 (ASE) \\
\hline Tephrosia purpurea (L.) Pers. & Erv & - & $\mathrm{NE}$ & Andrade-Lima, D s/n (ASE 1245) \\
\hline Zornia latifolia Sm. & Arb & - & $\mathrm{NE}$ & Córdula, E 144 (UFP) \\
\hline Zornia leptophylla (Benth.) Pittier & Arb & - & $\mathrm{NE}$ & Mendes, K 245 (UFP) \\
\hline Gentianaceae & & & & \\
\hline $\begin{array}{l}\text { Chelonanthus purpurascens (Aubl.) Struwe } \\
\text { et al. }\end{array}$ & Erv & - & $\mathrm{NE}$ & Vicente, A 53 (ASE) \\
\hline Coutoubea spicata Aubl. & Erv & - & $\mathrm{NE}$ & Amorim 180 (ASE) \\
\hline Curtia tenella (Mart.) Cham. & Erv & - & $\mathrm{NE}$ & Costa 432 (ASE) \\
\hline $\begin{array}{l}\text { Schultesia guianensis var. guianensis } \\
\text { (Aubl.) Malme }\end{array}$ & Erv & - & $\mathrm{NE}$ & Gomes 630 (ASE) \\
\hline Voyria obconica Progel & Erv-Sap & - & $\mathrm{NE}$ & Mendes et al. (2010) \\
\hline Gesneriaceae & & & & \\
\hline Codonanthe sp. & Erv & - & $\mathrm{NE}$ & Mendes et al. (2010) \\
\hline $\begin{array}{l}\text { Sinningia nordestina Chautems, Baracho \& } \\
\text { Siqueira-Filho } \\
\text { Heliconiaceae }\end{array}$ & Arb-Epi & - & $\mathrm{NE}$ & Amorim 141 (ASE) \\
\hline $\begin{array}{l}\text { Heliconia psittacorum L.f. } \\
\text { Humiriaceae }\end{array}$ & Erv & - & $\mathrm{NE}$ & Santos 432 (ASE) \\
\hline $\begin{array}{l}\text { Humiria balsamifera var. parvifolia (Juss.) } \\
\text { Cuatr. }\end{array}$ & Arb & - & $\mathrm{NE}$ & Fonseca, MR 629 (MOSS) \\
\hline $\begin{array}{l}\text { Sacoglottis mattogrossensis Malme } \\
\text { Hypericaceae }\end{array}$ & Arv & NR & $\mathrm{NE}$ & Alves-Araújo 964 (ASE) \\
\hline Vismia guianensis (Aubl.) Choisy & Arb & - & $\mathrm{NE}$ & Viana 345 (ASE) \\
\hline Hypoxidaceae & & & & \\
\hline Curculigo scorzonerifolia (Lam.) Baker & Erv & - & $\mathrm{LC}$ & Silva, FO 294 (ASE) \\
\hline $\begin{array}{l}\text { Hypoxis decumbens } \mathrm{L} . \\
\text { Iridaceae }\end{array}$ & Erv & - & $\mathrm{NE}$ & Lucena 1579 (ASE) \\
\hline Cipura paludosa Aubl. & Erv & - & $\mathrm{NE}$ & Araújo 2235 (ASE) \\
\hline Trimezia martinicensis (Jacq.) Herb. & Erv & - & $\mathrm{NE}$ & Lucena 1576 (ASE) \\
\hline
\end{tabular}


Tabela 1. Continuação.

\begin{tabular}{|c|c|c|c|c|}
\hline Família/Espécie & HAB & OCO & CAT & Coletor no./Autor(es) \\
\hline \multicolumn{5}{|l|}{ Krameriaceae } \\
\hline Krameria bahiana B. Simpson & Arb & - & $\mathrm{NE}$ & Dantas 17 (ASE) \\
\hline Krameria tomentosa A. St.-Hil. & Arb & - & LG & Costa 430 (ASE) \\
\hline \multicolumn{5}{|l|}{ Lamiaceae } \\
\hline Aegiphila pernambucensis Moldenke & Arb & NR & NE, EMA & Mendes, K 212 (UFP) \\
\hline Aegiphila verticillata Vell. & Arb & - & NE & Landim 665 (ASE) \\
\hline $\begin{array}{l}\text { Cyanocephalus rugosus (Benth.) Harley \& } \\
\text { J.F.B.Pastore }\end{array}$ & Erv & - & $\mathrm{NE}$ & Viana 1174 (ASE) \\
\hline $\begin{array}{l}\text { Eplingiella fruticosa (Salzm. ex Benth.) } \\
\text { Harley \& J.F.B. Pastore }\end{array}$ & Arb & - & $\mathrm{NE}$ & Fonseca, MR 407 (ASE) \\
\hline Hypenia salzmannii (Benth.) Harley & Erv & - & $\mathrm{NE}$ & Santos 48 (ASE) \\
\hline Hyptis atrorubens Poit. & Erv & - & $\mathrm{NE}$ & Santos 150 (ASE) \\
\hline Leonotis nepetifolia (L.) R.Br. & Erv & - & $\mathrm{NE}$ & Silva s/n (ASE 11593) \\
\hline Marsypianthes chamaedrys (Vahl) Kuntze & Arb & - & $\mathrm{NE}$ & Gomes 802 (ASE) \\
\hline Mesosphaerum pectinatum (L.) Kuntze & Arb & - & $\mathrm{NE}$ & Matos 60 (ASE) \\
\hline Rhaphiodon echinus Schauer & Arb & - & $\mathrm{NE}$ & Viana 1278 (ASE) \\
\hline Vitex rufescens A.Juss. & Arv & - & $\mathrm{NE}$ & Fonseca, MR 394 (ASE) \\
\hline \multicolumn{5}{|l|}{ Lauraceae } \\
\hline Aiouea saligna Miers & Arv & NR & $\mathrm{NE}$ & Landim 678 (ASE) \\
\hline Cassytha filiformis $\mathrm{L}$. & Erv-Sap & - & $\mathrm{NE}$ & Moura 763 (ASE) \\
\hline Ocotea fasciculata (Nees) Mez & Arv & NR & $\mathrm{NE}$ & Barreto s/n (ASE 526) \\
\hline Ocotea glomerata (Nees) Mez & Arv & - & $\mathrm{NE}$ & Viana s/n (ASE 5383) \\
\hline \multicolumn{5}{|l|}{ Lecythidaceae } \\
\hline Eschweilera ovata (Cambess.) Miers. & Arv & - & $\mathrm{NE}$ & Santos 108 (ASE) \\
\hline Lecythis lurida (Miers) S.A. Mori & Arv & - & LG & Mendes et al. (2010) \\
\hline Lecythis pisonis Cambess. & Arv & NR & $\mathrm{NE}$ & Matos 240 (ASE) \\
\hline \multicolumn{5}{|l|}{ Lentibulariaceae } \\
\hline Genlisea filiformis A. St.-Hil. & Erv & - & LC & Gomes 617 (ASE) \\
\hline $\begin{array}{l}\text { Utricularia amethystina Salmz. ex A. St.- } \\
\text { Hil. \& Girard }\end{array}$ & Erv & - & $\mathrm{NE}$ & Mendes 117 (ASE) \\
\hline Utricularia costata P.Taylor & Erv & - & $\mathrm{NE}$ & Carregosa 220 (ASE) \\
\hline Utricularia flaccida A.DC. & Erv & - & NT & Santos 394 (ASE) \\
\hline Utricularia longifolia Gardner & Erv & - & LC & Viana 1178 (ASE) \\
\hline Utricularia nana A.St.-Hil. \& Girard & Erv & - & $\mathrm{NE}$ & Carregosa 219 (ASE) \\
\hline Utricularia pusilla Vahl & Erv & - & $\mathrm{NE}$ & Landim, M 669 (HUEFS) \\
\hline Utricularia subulata L. & Erv & - & $\mathrm{NE}$ & Costa 382 (ASE) \\
\hline \multicolumn{5}{|l|}{ Linderniaceae } \\
\hline $\begin{array}{l}\text { Torenia thouarsii (Cham. \& } \text { Schltdl.) } \\
\text { Kuntze }\end{array}$ & Erv & - & $\mathrm{NE}$ & Santos 42 (ASE) \\
\hline \multicolumn{5}{|l|}{ Loganiaceae } \\
\hline Strychnos sp. & Arb & - & $\mathrm{NE}$ & Gomes 7 (ASE) \\
\hline \multicolumn{5}{|l|}{ Loranthaceae } \\
\hline Phthirusa sp. & Erv & - & $\mathrm{NE}$ & Mendes et al. (2010) \\
\hline Psittacanthus cordatus (Hoffmanns.) G.Don & Erv-Hpa & - & $\mathrm{NE}$ & Mendes et al. (2010) \\
\hline Psittacanthus dichroos (Mart.) Mart. & Erv-Hpa & - & $\mathrm{NE}$ & Landim 782 (ASE) \\
\hline Struthanthus concinnus (Mart.) Mart. & Erv-Hpa & NR & $\mathrm{NE}$ & Landim 892 (ASE) \\
\hline $\begin{array}{l}\text { Struthanthus syringifolius (Mart.) Mart. } \\
\text { Lythraceae }\end{array}$ & Erv-Hpa & - & $\mathrm{NE}$ & Matos 101 (ASE) \\
\hline Cuphea brachiata Martius ex Koehne & Arb & - & $\mathrm{NE}$ & Barros, F 2441 (CEN) \\
\hline Cuphea carthagenensis (Jacq.) J.F.Macbr. & Arb & - & NE & Nascimento-Junior 84 (ASE) \\
\hline Cuphea flava Spreng. & Arb & - & NE, EMA & Matos 237 (ASE) \\
\hline Cuphea linarioides Cham. \& Schltdl. & Arb & NR & LC & Santos 116 (ASE) \\
\hline Cuphea pulchra Moric. & Arb & - & $\mathrm{NE}$ & Lucena 1351 (ASE) \\
\hline Cuphea racemosa (L.f.) Spreng. & Arb & - & $\mathrm{NE}$ & Barreto s/n (ASE 24) \\
\hline Lafoensia pacari A.St.-Hil. & Arb & NR & LC & Campos 8 (ASE) \\
\hline \multicolumn{5}{|l|}{ Malpighiaceae } \\
\hline Byrsonima bahiana W.R. Anderson & Arb & - & NT, EMA & Landim 826 (ASE) \\
\hline Byrsonima blanchetiana Miq. & Arb & NR & LG & Melo 2 (ASE) \\
\hline Byrsonima chrysophylla Kunth & Arb & - & $\mathrm{NE}$ & Sousa, G $383(\mathrm{NY})$ \\
\hline Byrsonima coccolobifolia Kunth & Arb & - & $\mathrm{LC}$ & Nascimento-Junior s/n (ASE 11282) \\
\hline Byrsonima cydoniifolia A.Juss. & Arb & - & $\mathrm{NE}$ & Matos 211 (ASE) \\
\hline Byrsonima dealbata Griseb. & Arv & - & $\mathrm{NE}$ & Landim 693 (HUEFS) \\
\hline
\end{tabular}


Tabela 1. Continuação.

\begin{tabular}{|c|c|c|c|c|}
\hline Família/Espécie & HAB & $\mathrm{OCO}$ & CAT & Coletor no./Autor(es) \\
\hline Byrsonima gardneriana A.Juss. & Arb & - & $\mathrm{NE}$ & Silva, FO 236 (ASE) \\
\hline Byrsonima nitidifolia A. Juss. & Arb & NR & $\mathrm{NE}$ & Wassum, RA $8102(\mathrm{NY})$ \\
\hline Byrsonima sericea DC. & Arv & - & $\mathrm{NE}$ & Iran s/n (ASE 10884) \\
\hline Byrsonima verbascifolia (L.) DC. & Arb & - & $\mathrm{NE}$ & Gomes 230 (ASE) \\
\hline Heteropterys nordestina Amorim & Tre-L & - & $\mathrm{NE}$ & Viana 873 (ASE) \\
\hline Stigmaphyllon blanchetii C.E.Anderson & Tre-L & - & $\mathrm{NE}$ & Silva, FO 198 (ASE) \\
\hline Stigmaphyllon paralias A. Juss. & Tre-L & - & $\mathrm{NE}$ & Costa 431 (ASE) \\
\hline \multicolumn{5}{|l|}{ Malvaceae } \\
\hline Apeiba tibourbou Aubl. & Arv & - & $\mathrm{NE}$ & Prata 2904 (ASE) \\
\hline Corchorus argutus Kunth & Arb & NR & NE & Santos 112 (ASE) \\
\hline $\begin{array}{l}\text { Eriotheca macrophylla } \quad \text { (K.Schum.) } \\
\text { A.Robyns }\end{array}$ & Arv & NR & NE, EMA & Amorim 316 (ASE) \\
\hline Guazuma ulmifolia Lam. & Arv & - & $\mathrm{NE}$ & Vicente 114 (ASE) \\
\hline Pavonia cancellata (L.) Cav. & Arb & - & $\mathrm{NE}$ & Nascimento-Junior 193 (ASE) \\
\hline $\begin{array}{l}\text { Pavonia malacophylla (Link \& } \\
\text { Garcke }\end{array}$ & Arb & - & $\mathrm{NE}$ & Mendes et al. (2010) \\
\hline Sida angustissima A.St.-Hil. & Erv & - & $\mathrm{NE}$ & Esteves, Gl 2557 (SPF) \\
\hline Sida ciliaris $\mathrm{L}$. & Erv & - & $\mathrm{NE}$ & Nascimento-Junior 189 (ASE) \\
\hline Sida cordifolia L. & Arb & - & $\mathrm{NE}$ & Viana $1252(\mathrm{ASE})$ \\
\hline Sida linifolia Cav. & Arb & - & $\mathrm{NE}$ & Santos, ACAS 24 (ASE) \\
\hline Sida spinosa $\mathrm{L}$. & Erv & - & $\mathrm{NE}$ & Mendes et al. (2010) \\
\hline Sidastrum multiflorum (Jacq.) Fryxell & Arb & NR & $\mathrm{NE}$ & Prata 2876 (ASE) \\
\hline Sidastrum paniculatum (L.) Fryxell & Erv & - & $\mathrm{NE}$ & Santos 29 (ASE) \\
\hline Triumfetta rhomboidea Jacq. & Arb & NR & $\mathrm{NE}$ & Araujo 85 (ASE) \\
\hline Triumfetta semitriloba Jacq. & Arb & - & $\mathrm{NE}$ & Vicente 148 (ASE) \\
\hline Urena lobata L. & Erv & - & $\mathrm{NE}$ & Silva, FO 281 (ASE) \\
\hline Waltheria cinerascens A.St.-Hil. & Arb & NR & $\mathrm{NE}$ & Antonio s/n (ASE 12865) \\
\hline Waltheria indica $\mathrm{L}$ & Arb & - & NE & Mendes et al. (2010) \\
\hline Waltheria viscosissima A.St.-Hil. & Arb & NR & $\mathrm{NE}$ & Viana 1328 (ASE) \\
\hline \multicolumn{5}{|l|}{ Marantaceae } \\
\hline Goeppertia effusa Saka \& Lombardi & Erv & - & $\mathrm{NE}$ & Silva, FO 283 (ASE) \\
\hline Maranta noctiflora Regel \& Körn. & Erv & - & NE, EMA & Santos 434 (ASE) \\
\hline $\begin{array}{l}\text { Monotagma plurispicatum (Körn.) K. } \\
\text { Schum. }\end{array}$ & Erv & - & LC & Saka 221 (ASE) \\
\hline \multicolumn{5}{|l|}{ Marcgraviaceae } \\
\hline Norantea sp. & Arb & - & $\mathrm{NE}$ & Machado, WJ 1229 (ASE) \\
\hline $\begin{array}{l}\text { Schwartzia brasiliensis (Choisy) Bedell ex } \\
\text { Gir.-Cañas }\end{array}$ & Arb & - & $\mathrm{NE}$ & Araujo 1949 (ASE) \\
\hline \multicolumn{5}{|l|}{ Melastomataceae } \\
\hline Aciotis sp. & Erv & - & $\mathrm{NE}$ & Viana, G 183 (ASE) \\
\hline Acisanthera variabilis (Naud.) Triana & Erv & NR & $\mathrm{NE}$ & Mendes et al. (2010) \\
\hline Cambessedesia hilariana (Kunth) DC. & Erv & NR & LC & Barreto 37 (ASE) \\
\hline Cambessedesia tenuis Markgr. & Arb & NR & NE, ECA & Nascimento-Junior 143 (ASE) \\
\hline Clidemia capitellata (Bonpl.) D.Don & Arb & - & NE & Viana 354 (ASE) \\
\hline Clidemia debilis Crueg. & Arb & NR & $\mathrm{NE}$ & Mendes et al. (2010) \\
\hline Clidemia hirta (L.) D.Don & Erv & - & $\mathrm{NE}$ & Gomes 223 (ASE) \\
\hline Comolia ovalifolia (DC.) Triana & Arb & - & NE, EMA & Nascimento-Junior 78 (ASE) \\
\hline Comolia villosa (Aubl.) Triana & Erv & - & NE & Viana 1118 (ASE) \\
\hline Henriettea succosa (Aubl.) DC. & Arv & - & $\mathrm{NE}$ & Santos 20 (ASE) \\
\hline $\begin{array}{l}\text { Marcetia ericoides (Spreng.) O.Berg ex } \\
\text { Cogn. }\end{array}$ & Arb & - & $\mathrm{NE}$ & Valeria s/n (ASE 10857) \\
\hline Marcetia taxifolia (A.St.-Hl.) DC. & Arb & - & $\mathrm{NE}$ & Melo 3 (ASE) \\
\hline Miconia albicans (Sw.) Triana & Arb & - & NE & Matos 239 (ASE) \\
\hline Miconia amoena Triana & Arv & - & LC, EMA & Moura 767 (ASE) \\
\hline Miconia ciliata (Rich.) DC. & Arb & - & $\mathrm{NE}$ & Matos 231 (ASE) \\
\hline Miconia ferruginata DC. & Arb & - & $\mathrm{NE}$ & Costa 418 (ASE) \\
\hline Miconia francavillana Cogn. & Arb & - & NE, EMA & Vicente 196 (ASE) \\
\hline Miconia holosericea (L.) DC. & Arb & - & $\mathrm{NE}$ & Santos 123 (ASE) \\
\hline Miconia minutiflora (Bonpl.) DC. & Arb & - & $\mathrm{NE}$ & Vicente 5 (ASE) \\
\hline Miconia mirabilis (Aubl.) L.O.Williams & Arb & NR & $\mathrm{NE}$ & Matos, ECA 242 (ASE) \\
\hline Miconia prasina (Sw.) DC. & Arb & - & $\mathrm{NE}$ & Carneiro 473 (ASE) \\
\hline Nepsera aquatica (Aubl.) Naudin & Erv & - & $\mathrm{NE}$ & Santos 484 (ASE) \\
\hline
\end{tabular}


Tabela 1. Continuação.

\begin{tabular}{|c|c|c|c|c|}
\hline Família/Espécie & HAB & $\mathrm{OCO}$ & CAT & Coletor no./Autor(es) \\
\hline Ossaea sp. & Erv & - & $\mathrm{NE}$ & Melo 8 (ASE) \\
\hline Pterolepis cataphracta (Cham.) Triana & Erv & - & $\mathrm{NE}$ & Viana 1652 (ASE) \\
\hline Pterolepis glomerata (Rottb.) Miq. & Erv & - & LC & Viana 1086 (ASE) \\
\hline Pterolepis perpusilla (Naudin) Cogn. & Erv & - & LC & Landim 1050 (ASE) \\
\hline Pterolepis trichotoma (Rottb.) Cogn. & Erv & - & NE & Santos 46 (ASE) \\
\hline Tibouchina francavillana Cogn. & Arb & - & NE, EMA & Santana 662 (ASE) \\
\hline \multicolumn{5}{|l|}{ Meliaceae } \\
\hline Cedrela odorata $\mathrm{L}$. & Arv & - & VU & Fonseca 860 (ASE) \\
\hline Guarea guidonia (L.) Sleumer & Arv & - & $\mathrm{NE}$ & Fonseca 393 (ASE) \\
\hline Guarea macrophylla Vahl. & Arv & - & $\mathrm{NE}$ & Alves-Araújo 1121 (ASE) \\
\hline \multicolumn{5}{|l|}{ Menispermaceae } \\
\hline Cissampelos andromorpha DC. & Tre-H & - & $\mathrm{NE}$ & Gomes, LA 1127 (ASE) \\
\hline Cissampelos glaberrima A. St.-Hil. & Tre-H & NR & $\mathrm{NE}$ & Matos 168 (ASE) \\
\hline Cissampelos ovalifolia DC. & Tre-H & NR & $\mathrm{NE}$ & Costa s/n (ASE 14158) \\
\hline \multicolumn{5}{|l|}{ Microteaceae } \\
\hline $\begin{array}{l}\text { Microtea sp. } \\
\text { Moraceae }\end{array}$ & Erv & - & $\mathrm{NE}$ & Costa 344 (ASE) \\
\hline $\begin{array}{l}\text { Brosimum guianense (Aubl.) Huber ex } \\
\text { Ducke }\end{array}$ & Arv & - & $\mathrm{NE}$ & Mendes, K 370 (UFP) \\
\hline Ficus sp. & Arv & - & $\mathrm{NE}$ & Lima 129 (ASE) \\
\hline Sorocea hilarii Gaudich. & Arb & NR & $\mathrm{NE}$ & Vicente 138 (ASE) \\
\hline \multicolumn{5}{|l|}{ Myrtaceae } \\
\hline Calyptranthes brasiliensis Spreng. & Arv & - & $\mathrm{NE}$ & Vicente 85 (ASE) \\
\hline Calyptranthes clusiifolia O.Berg & Arv & - & $\mathrm{NE}$ & Araujo 1948 (ASE) \\
\hline Calyptranthes brasiliensis Spreng. & Arv & - & $\mathrm{NE}$ & Nascimento-Junior 217 (ASE) \\
\hline Campomanesia aromatica (Aubl.) Griseb. & Arb & NR & $\mathrm{LC}$ & Fonseca $857(\mathrm{ASE})$ \\
\hline Campomanesia ilhoensis Mattos & Arv & - & $\mathrm{NE}$ & Vicente 7 (ASE) \\
\hline Eugenia astringens Cambess. & Arv & NR & NE, EMA & Vicente 49 (ASE) \\
\hline Eugenia cerasiflora Miq. & Arv & - & LC & Vicente 145 (ASE) \\
\hline Eugenia punicifolia (Kunth) DC. & Arv & - & $\mathrm{NE}$ & Araujo 1957 (ASE) \\
\hline Marlierea excoriata Mart. & Arv & - & $\mathrm{NE}$ & Santana 150 (ASE) \\
\hline Myrcia amazonica DC. & Arv & - & $\mathrm{NE}$ & Viana 1653 (ASE) \\
\hline Myrcia decorticans DC. & Arv & - & $\mathrm{NE}$ & Dantas $32(\mathrm{ASE})$ \\
\hline Myrcia guianensis (Aubl.) DC. & Arv & NR & LC & Nascimento-Junior 114 (ASE) \\
\hline Myrcia hirtiflora DC. & Arv & NR & NE, EMA & Santos 126 (ASE) \\
\hline Myrcia ilheosensis Kiaersk. & Arv & NR & NE, EMA & Carneiro 477 (ASE) \\
\hline Myrcia multiflora (Lam.) DC. & Arv & NR & NE & Landim 694 (ASE) \\
\hline Myrcia neoblanchetiana E.Lucas \& Sobral & Arv & - & NE, EMA & Mendes et al. (2010) \\
\hline Myrcia polyantha DC. & Arv & - & $\mathrm{NE}$ & Campos 2 (ASE) \\
\hline Myrcia rosangelae NicLugh. & Arv & - & $\mathrm{NE}$ & Viana 1284 (ASE) \\
\hline Myrcia splendens (Sw.) DC. & Arb & - & $\mathrm{NE}$ & Lima 135 (ASE) \\
\hline Myrcia tomentosa (Aubl.) DC. & Arv & - & $\mathrm{NE}$ & Prata 2903 (ASE) \\
\hline $\begin{array}{l}\text { Myrciaria floribunda (H.West. ex Willd.) O. } \\
\text { Berg }\end{array}$ & & Viana 342 (ASE) \\
\hline Myrciaria tenella (DC.) O.Berg. & Arv & NR & DD & Araujo 1958 (ASE) \\
\hline Psidium decussatum DC. & Arv & - & $\mathrm{NE}$ & Silva 5 (ASE) \\
\hline Psidium guineense $\mathrm{Sw}$. & Arv & - & $\mathrm{NE}$ & Vicente 128 (ASE) \\
\hline Psidium oligospermum Mart. ex DC. & Arv & - & $\mathrm{NE}$ & Vicente 124 (ASE) \\
\hline \multicolumn{5}{|l|}{ Nyctaginaceae } \\
\hline Guapira noxia (Netto) Lundell & Arv & NR & $\mathrm{NE}$ & Prata 2888 (ASE) \\
\hline Guapira obtusata (Jacq.) Little & Arv & - & LC & Santana 51 (ASE) \\
\hline Guapira opposita (Vell.) Reitz & Arv & NR & NE & Vicente 109 (ASE) \\
\hline Guapira pernambucensis (Casar.) Lundell & Arb & - & NE, EMA & Matos 127 (ASE) \\
\hline Pisonia sp. & Arb & - & $\mathrm{NE}$ & Gomes 249 (ASE) \\
\hline \multicolumn{5}{|l|}{ Ochnaceae } \\
\hline Ouratea crassa Tiegh. & Arv & - & NE, EMA & Santana 48 (ASE) \\
\hline Ouratea cuspidata (A.St.-Hil.) Engl. & Arb & - & LC, EMA & Nascimento-Junior 224 (ASE) \\
\hline Ouratea hexasperma (A.St.-Hil.) Baill. & Arv & - & NE & Viana $1260(\mathrm{ASE})$ \\
\hline Ouratea parvifolia (A.St-Hil.) Engl. & Arv & NR & $\mathrm{NE}$ & Viana s/n (ASE 4784) \\
\hline Sauvagesia erecta $\mathrm{L}$. & Erv & - & $\mathrm{NE}$ & Costa 305 (ASE) \\
\hline \multicolumn{5}{|l|}{ Onagraceae } \\
\hline Ludwigia octovalvis (Jacg.) H.P. Raven & Erv & - & $\mathrm{NE}$ & Santos 130 (ASE) \\
\hline
\end{tabular}


Tabela 1. Continuação.

\begin{tabular}{|c|c|c|c|c|}
\hline Família/Espécie & HAB & $\mathrm{OCO}$ & CAT & Coletor no./Autor(es) \\
\hline \multicolumn{5}{|l|}{ Orchidaceae } \\
\hline Campylocentrum crassirhizum Hoehne & Erv-Epi & - & LC & Nascimento-Junior 145 (ASE) \\
\hline Catasetum discolor (Lindl.) Lindl. & Erv & - & $\mathrm{NE}$ & Santos 441 (ASE) \\
\hline Catasetum purum Nees \& Sinnings & Erv-Epi & - & LC, EMA & Mendes, K 146 (UFP) \\
\hline Catasetum uncatum Rolfe & Erv-Epi & - & NT, EMA & Santos 576 (ASE) \\
\hline \multicolumn{4}{|l|}{ Rchb.f. } & Nascimento-Junior 545 (ASE) \\
\hline Cyrtopodium parviflorum Lindl. & Erv & - & LC & Nascimento-Junior 236 (ASE) \\
\hline Dichaea panamensis Lindl. & Erv-Epi & - & $\mathrm{NE}$ & Mendes, K 344 (UFP) \\
\hline Encyclia alboxanthina Fowlie & Erv & - & $\mathrm{NE}$ & Landim 818 (ASE) \\
\hline Encyclia dichroma (Lindl.) Schltr. & Erv & - & NE, EMA & Nascimento-Junior 539 (ASE) \\
\hline Encyclia patens Hook. & Erv & - & NE & Viana $1175($ ASE) \\
\hline Epidendrum carpophorum Barb.Rodr. & Erv-Epi & - & LC & Landim 1396 (ASE) \\
\hline Epidendrum cinnabarinum Salzm. & Erv & - & $\mathrm{NE}$ & Santos 63 (ASE) \\
\hline Epidendrum orchidiflorum (Salzm.) Lindl. & Erv & - & $\mathrm{NE}$ & Carregosa 24 (ASE) \\
\hline Epidendrum secundum Jacq. & Erv & - & LC & Santos 490 (ASE) \\
\hline Epistephium lucidum Cogn. & Erv & - & $\mathrm{NE}$ & Mendes 283 (ASE) \\
\hline Galeandra montana Barb.Rodr. & Erv & - & $\mathrm{NE}$ & Monteiro 80 (ASE) \\
\hline \multicolumn{4}{|l|}{ N.H.Williams } & Landim, M 1032 (ASE) \\
\hline Habenaria fluminensis Hoehne & Erv & - & $\mathrm{NE}$ & Santos 183 (ASE) \\
\hline Habenaria hexaptera Lindl. & Erv & NR & LC & Santos, LAS 862 (ASE) \\
\hline Habenaria obtusa Lindl. & Erv & - & $\mathrm{NE}$ & Nascimento-Junior 134 (ASE) \\
\hline Habenaria parviflora Lindl. & Erv & - & $\mathrm{NE}$ & Santos 170 (ASE) \\
\hline Habenaria petalodes Lindl. & Erv & - & $\mathrm{NE}$ & Landim 688 (ASE) \\
\hline Habenaria rotundiloba Pabst & Erv & - & NE, EMA & Santos 570 (ASE) \\
\hline Habenaria trifida Kunth & Erv & - & LG & Carregosa 209 (ASE) \\
\hline Jacquiniella globosa (Jacq.) Schltr. & Erv-Epi & - & LC & Mendes, K 328 (UFP) \\
\hline Liparis nervosa (Thunb.) Lindl. & Erv & - & $\mathrm{NE}$ & Viana 1738 (ASE) \\
\hline Liparis vexillifera (La Llave \& Lex.) Cogn. & Erv & - & LC & Maciel, JR 329 (ASE) \\
\hline Oeceoclades maculata (Lindl.) Lindl. & Erv & - & $\mathrm{NE}$ & Prata 2776 (ASE) \\
\hline Polystachya estrellensis Rchb.f. & Erv-Epi & - & $\mathrm{NE}$ & Viana 1177 (ASE) \\
\hline Sacoila lanceolata (Aubl.) Garay & Erv & - & $\mathrm{NE}$ & Carregosa 26 (ASE) \\
\hline Sarcoglottis curvisepala Szlach. \& Rutk. & Erv & - & $\mathrm{NE}$ & Costa 473 (ASE) \\
\hline Scaphyglottis prolifera (R.Br.) Cogn. & Erv & NR & LC & Santos, LAS 572 (ASE) \\
\hline Scaphyglottis sickii Pabst & Erv-Epi & - & $\mathrm{NE}$ & Landim 1029 (ASE) \\
\hline Sobralia liliastrum Salzm. ex Lindl. & Erv & - & LC & Andrade 21 (ASE) \\
\hline Vanilla bahiana Hoehne & Tre-H & - & $\mathrm{NE}$ & Santos 449 (ASE) \\
\hline \multicolumn{5}{|l|}{ Orobanchaceae } \\
\hline Agalinis hispidula (Mart.) D'Arcy & Erv & - & $\mathrm{NE}$ & Matos, GMA 210 (ASE) \\
\hline Buchnera palustris (Aubl.) Spreng. & Erv & NR & $\mathrm{NE}$ & Barreto 69 (ASE) \\
\hline Esterhazya splendida J.C.Mikan & Arb & NR & $\mathrm{NE}$ & Schimdt, S 254 (UFP) \\
\hline \multicolumn{5}{|l|}{ Oxalidaceae } \\
\hline Oxalis divaricata Mart. ex Zucc. & Arb & - & $\mathrm{NE}$ & Viana 1836 (ASE) \\
\hline Oxalis frutescens $\mathrm{L}$. & Arb & - & $\mathrm{NE}$ & Fonseca 873 (ASE) \\
\hline Oxalis hedysarifolia Raddi & Arb & - & $\mathrm{LG}$ & Lucena, MFA 1770 (UFP) \\
\hline \multicolumn{5}{|l|}{ Passifloraceae } \\
\hline Passiflora alata Curtis & Tre-L & - & $\mathrm{NE}$ & Carneiro 478 (ASE) \\
\hline Passiflora cincinnata Mast. & Tre-L & - & $\mathrm{NE}$ & Araujo 104 (ASE) \\
\hline Passiflora contracta Vitta & Tre-L & NR & $\mathrm{NE}$ & Araujo 2240 (ASE) \\
\hline Passiflora edulis Sims & Tre-L & - & LC & Araujo 103 (ASE) \\
\hline Passiflora luetzelburgii Harms & Tre-L & - & $\mathrm{NE}$ & Santos, LAS 701 (ASE) \\
\hline Passiflora miersii Mart. & Tre-L & - & LC & Carneiro 476 (ASE) \\
\hline Passiflora misera Kunth & Tre-H & - & $\mathrm{NE}$ & Lucena 1815 (ASE) \\
\hline Passiflora silvestris Vell. & Tre-H & - & $\mathrm{NE}$ & Santos 5 (ASE) \\
\hline Passiflora watsoniana Mast. & Tre-H & NR & NE, EMA & Mendes et al. (2010) \\
\hline \multicolumn{5}{|l|}{ Peraceae } \\
\hline Chaetocarpus myrsinites Baill. & Arv & - & $\mathrm{NE}$ & Alves-Araujo 1117 (ASE) \\
\hline Pera glabrata (Schott) Poepp. ex Baill. & Arv & - & $\mathrm{NE}$ & Santos 460 (ASE) \\
\hline $\begin{array}{l}\text { Pogonophora schomburgkiana Miers ex } \\
\text { Benth. }\end{array}$ & Arv & - & $\mathrm{NE}$ & Lucena et al. (2009) \\
\hline
\end{tabular}


Tabela 1. Continuação.

\begin{tabular}{|c|c|c|c|c|}
\hline Família/Espécie & $\overline{\mathrm{HAB}}$ & OCO & CAT & Coletor no./Autor(es) \\
\hline \multicolumn{5}{|l|}{ Phyllanthaceae } \\
\hline Phyllanthus flagelliformis Müll.Arg. & Arb & NR & $\mathrm{NE}$ & Lucena et al. (2009) \\
\hline Phyllanthus heteradenius Müll.Arg. & Arb & - & $\mathrm{NE}$ & Lucena et al. (2009) \\
\hline Richeria grandis Vahl & Arv & - & $\mathrm{NE}$ & Viana 94 (ASE) \\
\hline \multicolumn{5}{|l|}{ Piperaceae } \\
\hline Peperomia glabella (Sw.) A.Dietr. & Erv-Epi & NR & $\mathrm{NE}$ & Silva, FO 262 (ASE) \\
\hline Peperomia magnoliifolia (Jacq.) A.Dietr. & Erv & - & $\mathrm{NE}$ & Mendes 315 (ASE) \\
\hline Piper arboreum Aubl. & Arb & - & $\mathrm{NE}$ & Mendes 338 (ASE) \\
\hline Piper hispidum Sw. & Erv & - & $\mathrm{NE}$ & Santana, JP 138 (ASE) \\
\hline Piper ilheusense Yunck. & Arb & NR & NE, EMA & Mendes et al. (2010) \\
\hline Piper klotzschianum (Kunth) C.DC. & Arb & NR & NE & Santana, JP 136 (ASE) \\
\hline Piper tuberculatum Jacq. & Arb & NR & NE & Vicente 170 (ASE) \\
\hline Piper vicosanum Yunck. & Arb & NR & LC & Gomes 651 (ASE) \\
\hline \multicolumn{5}{|l|}{ Plantaginaceae } \\
\hline Angelonia biflora Benth. & Erv & NR & NE, ECA & Barreto 45 (ASE) \\
\hline Angelonia cornigera Hook.f. & Erv & - & NE & Dantas 71 (ASE) \\
\hline Angelonia salicariifolia Bonpl. & Erv & - & $\mathrm{NE}$ & Mendes et al. (2010) \\
\hline Scoparia dulcis $\mathrm{L}$. & Arb & - & $\mathrm{NE}$ & Santos 142 (ASE) \\
\hline Stemodia foliosa Benth. & Arb & - & $\mathrm{NE}$ & Gomes 21 (ASE) \\
\hline \multicolumn{5}{|l|}{ Poaceae } \\
\hline Andropogon bicornis $\mathrm{L}$. & Erv & - & $\mathrm{NE}$ & Santos 440 (ASE) \\
\hline Andropogon ingratus var. ingratus Hack. & Erv & - & $\mathrm{NE}$ & Santos 97 (ASE) \\
\hline Andropogon leucostachyus Kunth & Erv & - & $\mathrm{NE}$ & Dantas 106 (ASE) \\
\hline Andropogon selloanus (Hack.) Hack. & Erv & - & $\mathrm{NE}$ & Viana, G 1130 (IMA) \\
\hline Andropogon virgatus Desv. & Erv & - & $\mathrm{NE}$ & Viana 1127 (ASE) \\
\hline $\begin{array}{l}\text { Apochloa lutzii (Swallen) Zuloaga \& } \\
\text { Morrone }\end{array}$ & Erv & NR & NE, EMA & Carregosa 59 (ASE) \\
\hline Aristida longifolia Trin. & Erv & - & $\mathrm{NE}$ & Maciel, JR 271 (UFP) \\
\hline Aristida setifolia Kunth & Erv & NR & $\mathrm{NE}$ & Viana 1320 (ASE) \\
\hline Atractantha falcata McClure & Erv & NR & NE, EMA & Santos 95 (ASE) \\
\hline Axonopus aureus P.Beauv. & Erv & - & NE & Dantas 30 (ASE) \\
\hline Axonopus capillaris (Lam.) Chase & Erv & - & $\mathrm{NE}$ & Maciel \& Alves (2011) \\
\hline Axonopus conduplicatus G.A. Black & Erv & - & $\mathrm{NE}$ & Dantas 108 (ASE) \\
\hline Axonopus laxiflorus (Trin.) Chase & Erv & NR & $\mathrm{NE}$ & Maciel \& Alves (2011) \\
\hline $\begin{array}{l}\text { Axonopus pellitus (Nees ex Trin.) Hitchc. } \\
\& \text { Chase }\end{array}$ & Erv & NR & $\mathrm{NE}$ & Santos 141 (ASE) \\
\hline Axonopus polydactylus (Steud.) Dedecca & Erv & - & $\mathrm{NE}$ & Santos 86 (ASE) \\
\hline Axonopus purpusii (Mez) Chase & Erv & - & $\mathrm{NE}$ & Maciel \& Alves (2011) \\
\hline Cenchrus echinatus $\mathrm{L}$. & Erv & NR & $\mathrm{NE}$ & Borba s/n (ASE 15605) \\
\hline Chloris barbata Sw. & Erv & - & $\mathrm{NE}$ & Dantas 104 (ASE) \\
\hline Echinolaena inflexa (Poir.) Chase & Erv & - & $\mathrm{NE}$ & Costa 324 (ASE) \\
\hline Eleusine indica (L.) Gaertn. & Erv & - & $\mathrm{NE}$ & Dantas 112 (ASE) \\
\hline Eragrostis articulata (Schrank.) Nees & Erv & - & $\mathrm{NE}$ & Dantas 20 (ASE) \\
\hline Eragrostis bahiensis Schrad. ex Schult. & Erv & NR & $\mathrm{NE}$ & Dantas 116 (ASE) \\
\hline Eragrostis ciliaris (L.) R.Br. & Erv & - & $\mathrm{NE}$ & Nascimento-Junior 133 (ASE) \\
\hline Eragrostis maypurensis (Kunth) Steud. & Erv & NR & $\mathrm{NE}$ & Carregosa 60 (ASE) \\
\hline Eragrostis rufescens Schrad. ex Schult. & Erv & - & $\mathrm{NE}$ & Matos 6 (ASE) \\
\hline Eragrostis secundiflora J.Presl & Erv & - & $\mathrm{NE}$ & Costa 505 (ASE) \\
\hline Gymnopogon foliosus (Willd.) Nees & Erv & NR & $\mathrm{NE}$ & Landim 723 (ASE) \\
\hline Homolepis isocalycia (G.Mey) Chase & Erv & - & $\mathrm{NE}$ & Rocha-Santos 1 (ASE) \\
\hline Ichnanthus calvescens (Nees ex Trin.) Döll & Erv & - & $\mathrm{NE}$ & Nascimento-Junior 87 (ASE) \\
\hline Ichnanthus dasycoleus Tutin & Erv & - & $\mathrm{NE}$ & Gomes, P 649 (UFP) \\
\hline Ichnanthus lancifolius $\mathrm{Mez}$ & Erv & NR & NE, EMA & Maciel \& Alves (2011) \\
\hline $\begin{array}{l}\text { Ichnanthus nemoralis (Schrad.) Hitchc. \& } \\
\text { Chase }\end{array}$ & Erv & - & NE & Maciel, JR 295 (UFP) \\
\hline Lasiacis divaricata (L.) Hitchc. & Erv & - & $\mathrm{NE}$ & Santos 62 (ASE) \\
\hline $\begin{array}{l}\text { Lasiacis sorghoidea (Desv. ex Ham.) } \\
\text { Hitchc. \& Chase }\end{array}$ & Erv & - & $\mathrm{NE}$ & Matos 52 (ASE) \\
\hline Melinis repens (Willd.) Zizka & Erv & NR & $\mathrm{NE}$ & Nascimento-Junior 593 (ASE) \\
\hline Mesosetum loliiforme (Hochst.) Chase & Erv & - & $\mathrm{NE}$ & Dantas 120 (ASE) \\
\hline $\begin{array}{l}\text { Ocellochloa soderstromii (Zuloaga \& } \\
\text { Send.) Zuloaga \& Morrone ex Filg \& Rodr }\end{array}$ & Erv & NR & $\mathrm{NE}$ & Santos 80 (ASE) \\
\hline
\end{tabular}


Tabela 1. Continuação.

\begin{tabular}{|c|c|c|c|c|}
\hline Família/Espécie & HAB & OCO & CAT & Coletor no./Autor(es) \\
\hline Panicum trichoides $\mathrm{Sw}$. & Erv & - & $\mathrm{NE}$ & Matos, E 63 (ASE) \\
\hline Pappophorum pappiferum (Lam.) Kuntze & Erv & NR & $\mathrm{NE}$ & Dantas, T 101 (ASE) \\
\hline $\begin{array}{l}\text { Parodiolyra micrantha (Kunth) Davidse \& } \\
\text { Zuloaga }\end{array}$ & Erv & - & $\mathrm{NE}$ & Prata 2786A (ASE) \\
\hline Paspalidium geminatum (Forssk.) Stapf & Erv & NR & $\mathrm{NE}$ & Santos 95 (ASE) \\
\hline Paspalum arenarium Schrad. & Erv & - & $\mathrm{NE}$ & Viana 1138 (ASE) \\
\hline $\begin{array}{l}\text { Paspalum convexum Humb. \& Bonpl. ex } \\
\text { Flüggé }\end{array}$ & Erv & - & $\mathrm{NE}$ & Maciel 270 (ASE) \\
\hline Paspalum divergens Döll & Erv & - & NE, EMA & Landim 725 (ASE) \\
\hline Paspalum gardnerianum Nees & Erv & - & $\mathrm{NE}$ & Maciel 306 (UFP) \\
\hline Paspalum ligulare Nees & Erv & NR & $\mathrm{NE}$ & Dantas 111 (ASE) \\
\hline Paspalum maritimum Trin. & Erv & NR & $\mathrm{NE}$ & Santos, ML 103 (ASE) \\
\hline Paspalum melanospermum Desv. ex Poir. & Erv & - & $\mathrm{NE}$ & Almeida, ES 360 (ASE) \\
\hline Paspalum millegrana Schrad. ex Schult. & Erv & - & $\mathrm{NE}$ & Dantas 110 (ASE) \\
\hline Paspalum pulchellum Kunth & Erv & - & $\mathrm{NE}$ & Viana 2125 (ASE) \\
\hline Paspalum scutatum Nees ex Trin. & Erv & - & NE, ECA & Maciel, JR 277 (UFP) \\
\hline Plagiantha tenella Renvoize & Erv & NR & NE & Dantas 115 (ASE) \\
\hline Rhytachne rottboellioides Desv. ex Ham. & Erv & NR & $\mathrm{NE}$ & Dantas 114 (ASE) \\
\hline Rugoloa pilosa (Sw.) Zuloaga & Erv & - & $\mathrm{NE}$ & Santos, ML 52 (ASE) \\
\hline Schizachyrium condensatum (Kunth) Nees & Erv & NR & $\mathrm{NE}$ & Matos, E 48 (ASE) \\
\hline Setaria tenax (Rich.) Desv. & Erv & - & NE & Carneiro 304 (ASE) \\
\hline Sporobolus ciliatus J.Presl. & Erv & NR & $\mathrm{NE}$ & Maciel, JR 265 (UFP) \\
\hline Sporobolus cubensis Hitchc. & Erv & - & $\mathrm{NE}$ & Dantas 113 (ASE) \\
\hline Sporobolus indicus (L.) R.Br. & Erv & - & $\mathrm{NE}$ & Dantas 119 (ASE) \\
\hline Sporobolus tenuissimus (Schrank) Kuntze & Erv & NR & $\mathrm{NE}$ & Maciel \& Alves (2011) \\
\hline Steinchisma laxum (Sw.) Zuloaga & Erv & - & $\mathrm{NE}$ & Santos, L 79 (ASE) \\
\hline Trachypogon spicatus (L.f.) Kuntze & Erv & NR & $\mathrm{NE}$ & Dantas 81 (ASE) \\
\hline Trichanthecium cyanescens (Nees ex Trin.) & Erv & - & $\mathrm{NE}$ & Maciel \& Alves (2011) \\
\hline Zuloaga \& Morrone & & & & \\
\hline Trichanthecium parvifolium (Lam.) & Erv & - & $\mathrm{NE}$ & Santos, ML 27 (ASE) \\
\hline Zuloaga \& Morrone & & & & \\
\hline $\begin{array}{l}\text { Trichanthecium polycomum } \\
\text { Zuloaga \& Morrone }\end{array}$ & Erv & - & $\mathrm{NE}$ & Maciel \& Alves (2011) \\
\hline Polygalaceae & & & & \\
\hline Asemeia martiana & Erv & - & $\mathrm{NE}$ & Córdula, E 136 (UFP) \\
\hline J.F.B.Pastore \& J.R.Abbott & & & & \\
\hline $\begin{array}{l}\text { Asemeia violacea (Aubl.) J.F.B.Pastore \& } \\
\text { J.R.Abbott }\end{array}$ & Erv & - & $\mathrm{NE}$ & Schmidt 271 (ASE) \\
\hline $\begin{array}{l}\text { Bredemeyera laurifolia (A.St.-Hil.) Klotzsch } \\
\text { ex A.W. Benn. }\end{array}$ & Erv & - & $\mathrm{NE}$ & Viana 1718 (ASE) \\
\hline Polygala galioides Poir. & Erv & - & LC & Viana 1977 (ASE) \\
\hline Polygala glochidata Kunth & Erv & - & $\mathrm{NE}$ & Viana 1243 (ASE) \\
\hline Polygala longicaulis Kunth & Erv & - & $\mathrm{NE}$ & Viana 1727 (ASE) \\
\hline Polygala trichosperma Jacq. & Erv & - & $\mathrm{NE}$ & Landim, M 714 (ASE) \\
\hline Polygonaceae & & & & \\
\hline Coccoloba laevis Casar. & Arv & - & NE, EMA & Dantas, T 2 (ASE) \\
\hline Coccoloba lucidula Benth. & Arv & - & NE & Lucena, MFA 1762 (UFP) \\
\hline Coccoloba mollis Casar. & Arv & - & $\mathrm{NE}$ & Vicente 158 (ASE) \\
\hline Coccoloba parimensis Benth. & Arv & - & $\mathrm{NE}$ & Araujo 1950 (ASE) \\
\hline Coccoloba rosea Meisn. & Arv & - & NE, EMA & Schmidt 283 (ASE) \\
\hline Pontederiaceae & & & & \\
\hline Eichhornia crassipes (Mart.) Solms & Erv & - & $\mathrm{NE}$ & Nogueira, PCL s/n (ASE 25631) \\
\hline Portulacaceae & & & & \\
\hline Portulaca halimoides $\mathrm{L}$. & Erv & - & LC & Prata 2770 (ASE) \\
\hline Primulaceae & & & & \\
\hline Myrsine guianensis (Aubl.) Kuntze & Arv & - & $\mathrm{NE}$ & Vicente 146 (IPA) \\
\hline Myrsine umbellata Mart. & Arv & - & $\mathrm{NE}$ & Wasum, RA s/n (NY 495886) \\
\hline Rhamnaceae & & & & \\
\hline Gouania blanchetiana Miq. & Tre-L & - & $\mathrm{LC}$ & Landim 697 (ASE) \\
\hline Rubiaceae & & & & \\
\hline Alibertia sp. & Arv & - & $\mathrm{NE}$ & Dantas 89 (ASE) \\
\hline Alseis floribunda Schott & Arb & - & $\mathrm{NE}$ & Santos, L 476 (ASE) \\
\hline
\end{tabular}


Tabela 1. Continuação.

\begin{tabular}{|c|c|c|c|c|c|}
\hline Família/Espécie & HAB & OCO & CAT & Coletor no./Autor(es) & \\
\hline Borreria capitata (Ruiz \& Pav.) DC. & Erv & - & $\mathrm{NE}$ & Dantas 49 (ASE) & \\
\hline Borreria humifusa Mart. & Erv & - & NE, EMA & Nascimento-Junior 128 (ASE) & \\
\hline Borreria spinosa Cham. et Schltdl. & Erv & - & $\mathrm{NE}$ & Santos, ACAS 27 (ASE) & \\
\hline Borreria verticillata (L.) G.Mey & Erv & NR & $\mathrm{NE}$ & Costa 469 (ASE) & \\
\hline Chiococca nitida Benth. & Arb & - & $\mathrm{NE}$ & Araujo 1953 (ASE) & \\
\hline Chiococca plowmanii Delprete & Arv & - & NE, EMA & Fonseca s/n (ASE 214) & \\
\hline Chomelia obtusa Cham. \& Schltdl. & Arb & - & NE & Vicente 154 (ASE) & \\
\hline $\begin{array}{l}\text { Cordiera myrciifolia (K.Schum.) C.H.Perss. } \\
\text { \& Delprete }\end{array}$ & Arb & NR & $\mathrm{NE}$ & Santos, ML 66 (ASE) & \\
\hline Cordiera obtusa (K.Schum) Kuntze. & Arv & NR & $\mathrm{NE}$ & Viana 1137 (ASE) & \\
\hline Coutarea hexandra (Jacq.) K.Schum. & Arv & - & $\mathrm{NE}$ & Prata, AP 2868 (ASE) & \\
\hline Declieuxia aspalathoides Müll.Arg. & Erv & - & $\mathrm{NE}$ & Carregosa, T 14 (ASE) & \\
\hline $\begin{array}{l}\text { Declieuxia fruticosa (Willd. ex Roem \& } \\
\text { Schult.) Kuntze }\end{array}$ & Arb & - & LC & Nascimento-Junior 230 (ASE) & \\
\hline $\begin{array}{l}\text { Emmeorhiza umbellata (Spreng.) K. } \\
\text { Schum. }\end{array}$ & Tre-L & - & $\mathrm{NE}$ & Santos, ACAS 41 (ASE) & \\
\hline Esterhazya splendida J.C.Mikan & Sub & NR & $\mathrm{NE}$ & Silva, FO 163 (ASE) & \\
\hline Geophila repens (L.) I.M. Johnst. & Erv & NR & $\mathrm{NE}$ & Prata, AP 2897 (ASE) & \\
\hline Guettarda platypoda DC. & Arb & - & NE & Barreto s/n (ASE 569) & \\
\hline Guettarda sericea Müll.Arg. & Arb & - & NE, ECA & Vicente 132 (ASE) & \\
\hline Guettarda viburnoides Cham. \& Schltdl. & Arv & NR & NE & Vicente 168 (ASE) & \\
\hline $\begin{array}{l}\text { Hexasepalum apiculatum (Willd.) Delprete } \\
\& \text { J.H. Kirkbr. }\end{array}$ & Arb & - & $\mathrm{NE}$ & Costa, SM 423 (ASE) & \\
\hline Hexasepalum teres (Walter) J.H. Kirkbr. & Erv & - & $\mathrm{NE}$ & Silva, FO 119 (ASE) & \\
\hline Leptoscela ruellioides Hook.f. & Erv & - & $\mathrm{NE}$ & Carneiro, EM 295 (ASE) & \\
\hline $\begin{array}{l}\text { Margaritopsis chaenotricha } \\
\text { C.M.Taylor }\end{array}$ & Arb & - & NE, EMA & Gomes, P 773 (UFP) & \\
\hline $\begin{array}{l}\text { Mitracarpus frigidus (Willd. ex Roem. \& } \\
\text { Schult.) K. Schum. }\end{array}$ & Arb & NR & $\mathrm{NE}$ & Landim 682 (ASE) & \\
\hline $\begin{array}{l}\text { Mitracarpus polygonifolius (A. St.-Hil.) } \\
\text { R.M. Salas \& E.B. Souza }\end{array}$ & Arb & - & $\mathrm{NE}$ & Matos, ECA 26 (ASE) & \\
\hline $\begin{array}{l}\text { Mitracarpus strigosus (Thunb.) P.L.R. } \\
\text { Moraes, De Smedt \& Hjertson }\end{array}$ & Erv & - & $\mathrm{NE}$ & Matos, ECA 31 (ASE) & \\
\hline Oldenlandia filicaulis K.Schum. & Erv & - & $\mathrm{NE}$ & Landim 672 (ASE) & \\
\hline Palicourea marcgravii A.St.-Hil. & Arb & - & $\mathrm{NE}$ & Santos, ML 137 (ASE) & \\
\hline Perama hirsuta Aubl. & Erv & - & $\mathrm{NE}$ & Nascimento-Junior 80 (ASE) & \\
\hline Psychotria bracteocardia (DC.) Müll. Arg. & Arb & - & $\mathrm{NE}$ & Santos, ML 44 (ASE) & \\
\hline Psychotria capitata Ruiz \& Pavan & Arb & - & LC & Ribeiro, A410 (HUEFS) & \\
\hline Psychotria carthagenensis Jacq. & Arb & - & $\mathrm{NE}$ & Santos, ML 68 (ASE) & \\
\hline $\begin{array}{l}\text { Psychotria hoffmannseggiana (Willd. ex } \\
\text { Schult.) Müll. Arg. }\end{array}$ & Arb & - & $\mathrm{NE}$ & Carneiro 363 (ASE) & \\
\hline Randia armata (Sw.) DC. & Arb & - & $\mathrm{NE}$ & Fonseca, MR 854 (ASE) & \\
\hline $\begin{array}{l}\text { Richardia grandiflora (Cham. \& Schltdl.) } \\
\text { Steud. }\end{array}$ & Erv & - & $\mathrm{NE}$ & Rocha, RM s/n (ASE 26331) & \\
\hline Salzmannia nitida DC. & Arb & - & NE, EMA & $\begin{array}{l}\text { Nascimento-Junior } \quad \mathrm{s} / \mathrm{n} \\
11284 \text { ) }\end{array}$ & (ASE \\
\hline Spermacoce decipiens (K.Schum.) Kuntze & Erv & - & RAR, NE & Dantas 65 (ASE) & \\
\hline $\begin{array}{l}\text { Staelia virgata (Link ex Roem. \& Schult.) } \\
\text { K.Schum. }\end{array}$ & Sub & - & NE & Silva, FO 107 (ASE) & \\
\hline $\begin{array}{l}\text { Tocoyena formosa (Cham. \& Schltdl.) K. } \\
\text { Schum. } \\
\text { Rutaceae }\end{array}$ & Arv & - & $\mathrm{NE}$ & Prata 2867 (ASE) & \\
\hline Conchocarpus insignis Pirani & Arv & NR & NE, EMA & Alves-Araujo, A 977 (UFP) & \\
\hline Ertela trifolia (L.) Kuntze & Erv & - & NE & Prata 2895 (ASE) & \\
\hline Esenbeckia grandiflora Mart. & Arb & - & $\mathrm{NE}$ & Dantas, TV 46 (ASE) & \\
\hline Salicaceae & & & & & \\
\hline Casearia grandiflora Cambess. & Arv & - & $\mathrm{NE}$ & Matos, GMA 172 (ASE) & \\
\hline $\begin{array}{l}\text { Casearia sylvestris } \mathrm{Sw} . \\
\text { Santalaceae }\end{array}$ & Arb & - & $\mathrm{NE}$ & Vicente 149 (ASE) & \\
\hline Phoradendron chrysocladon A.Gray & Erv-Hpa & - & $\mathrm{NE}$ & Santana, MC 141 (ASE) & \\
\hline Phoradendron strongyloclados Eichler & Erv-Hpa & - & $\mathrm{NE}$ & Dantas, TVP 42 (ASE) & \\
\hline
\end{tabular}


Tabela 1. Continuação.

\begin{tabular}{|c|c|c|c|c|}
\hline Família/Espécie & HAB & OCO & GAT & Coletor no./Autor(es) \\
\hline \multicolumn{5}{|l|}{ Sapindaceae } \\
\hline $\begin{array}{l}\text { Allophylus edulis (A.St.-Hil. et al.) Hieron. } \\
\text { ex Niederl. }\end{array}$ & Arv & - & $\mathrm{NE}$ & Vicente 135 (ASE) \\
\hline Cupania impressinervia Acev.-Rodr. & Arb & - & $\mathrm{NE}$ & Prata 2898 (ASE) \\
\hline Paullinia trigonia Vell. & Tre-L & - & NE & Gomes, E 227 (ASE) \\
\hline Paullinia weinmanniifolia Mart. & Tre-L & - & NE, EMA & Mendes et al. (2010) \\
\hline Serjania communis Cambess. & Tre-L & NR & NE & Viana 348 (ASE) \\
\hline Serjania salzmanniana Schltdl. & Tre-L & - & $\mathrm{NE}$ & Landim 888 (ASE) \\
\hline Talisia esculenta (Cambess.) Radlk. & Arv & - & $\mathrm{NE}$ & Fonseca, MR 865 (ASE) \\
\hline \multicolumn{5}{|l|}{ Sapotaceae } \\
\hline Manilkara rufula (Miq.) H.J. Lam & Arv & - & LC & Fonseca, MR 406 (ASE) \\
\hline Manilkara salzmannii (A.DC.) H.J.Lam & Arv & - & $\mathrm{NE}$ & Barreto, ACC s/n (ASE 383) \\
\hline Pouteria gardneri (Mart. \& Miq.) Baehni & Arv & - & $\mathrm{NE}$ & Alves-Araujo, A 1116 (UFP) \\
\hline Pouteria macahensis T.D. Penn. & Arv & - & EN, EMA & Matos, ECA 244 (ASE) \\
\hline Pradosia sp. & Arv & - & NE & Santos, ML 12 (ASE) \\
\hline \multicolumn{5}{|l|}{ Schoepfiaceae } \\
\hline Schoepfia brasiliensis A.DC. & Arv & - & NE & Viana 1810 (ASE) \\
\hline \multicolumn{5}{|l|}{ Simaroubaceae } \\
\hline Simarouba amara Aubl. & Arv & - & $\mathrm{NE}$ & Paula 3640 (ASE) \\
\hline \multicolumn{5}{|l|}{ Siparunaceae } \\
\hline Siparuna guianensis Aubl. & Arb & - & $\mathrm{NE}$ & Vicente, A 117 (ASE) \\
\hline \multicolumn{5}{|l|}{ Smilacaceae } \\
\hline Smilax rufescens Griseb. & Tre-L & NR & $\mathrm{NE}$ & Matos, ECA 38 (ASE) \\
\hline \multicolumn{5}{|l|}{ Solanaceae } \\
\hline Aureliana fasciculata (Vell.) Sendtn. & Arb & - & LC & Vicente, A 141 (ASE) \\
\hline Cestrum axillare Vell. & Arb & - & $\mathrm{NE}$ & Vicente, A 193 (ASE) \\
\hline Schwenckia americana Rooyen ex L. & Arb & - & $\mathrm{NE}$ & Alves-Araujo, A 1108 (UFP) \\
\hline Solanum americanum Mill. & Arb & - & $\mathrm{NE}$ & Matos, ECA 7 (ASE) \\
\hline Solanum asperum Rich. & Arb & - & $\mathrm{NE}$ & Viana, G 1257 (CEPEC) \\
\hline Solanum asterophorum Mart. & Arb & - & NE, EMA & Matos, GMA 170 (ASE) \\
\hline Solanum megalonyx Sendtn. & Arb & - & $\mathrm{NE}$ & Vicente, A 126 (ASE) \\
\hline Solanum paludosum Moric. & Arb & - & $\mathrm{NE}$ & Prata, 2900 (ASE) \\
\hline $\begin{array}{l}\text { Solanum stipulaceum Willd. ex Roem. \& } \\
\text { Schult. }\end{array}$ & Arb & - & $\mathrm{NE}$ & Fontenelle, 10 (ASE) \\
\hline \multicolumn{5}{|l|}{ Thelypteridaceae } \\
\hline Thelypteris sp. & Erv & - & $\mathrm{NE}$ & Santiago, A 1311 (ASE) \\
\hline \multicolumn{5}{|l|}{ Trigoniaceae } \\
\hline Trigonia nivea var. nivea Cambess & Arb & - & $\mathrm{NE}$ & Prata, 2916 (ASE) \\
\hline \multicolumn{5}{|l|}{ Turneraceae } \\
\hline $\begin{array}{l}\text { Piriqueta duarteana var. duarteana } \\
\text { (Cambess.) Urb. }\end{array}$ & Arb & - & $\mathrm{NE}$ & Costa, 354 (ASE) \\
\hline $\begin{array}{l}\text { Piriqueta guianensis subsp. elongata (Urb.) } \\
\text { Arbo }\end{array}$ & Arb & - & $\mathrm{NE}$ & Viana, 830 (ASE) \\
\hline Turnera chamaedrifolia Cambess. & Arb & - & $\mathrm{NE}$ & Santos, LAS 457 (ASE) \\
\hline Turnera coerulea var. coerulea DC. & Arb & - & $\mathrm{NE}$ & Fonseca, MR s/n (ASE) \\
\hline Turnera coerulea var. surinamensis (Urb.) & Arb & - & $\mathrm{NE}$ & Santos, ML 147 (ASE) \\
\hline \multicolumn{5}{|l|}{ Arbo \& Fernández } \\
\hline Turnera hermannioides Cambess & Arb & - & $\mathrm{NE}$ & Menezes, AB 75 (ASE) \\
\hline Turnera melochioides Cambess. & Arb & - & $\mathrm{NE}$ & Costa, SM 354 (ASE) \\
\hline \multicolumn{5}{|l|}{ Urticaceae } \\
\hline Cecropia pachystachya Trécul & Arv & - & $\mathrm{NE}$ & Gomes, P 623 (ASE) \\
\hline Cecropia palmata Willd. & Arv & - & $\mathrm{NE}$ & Thomas, WW 8903 (NY) \\
\hline Urera baccifera (L.) Gaudich ex Wedd. & Arb & - & $\mathrm{NE}$ & Vicente, A 137 (ASE) \\
\hline Urera caracasana (Jacq.) Griseb. & Arb & - & $\mathrm{NE}$ & Vicente, A 139 (ASE) \\
\hline \multicolumn{5}{|l|}{ Velloziaceae } \\
\hline $\begin{array}{l}\text { Vellozia cinerascens (Mart. ex Schult. f.) } \\
\text { Mart. ex Schult. f. }\end{array}$ & Erv & NR & NE, ECA & Carneiro, E 323 (ASE) \\
\hline Vellozia dasypus Seub. & Erv & - & $\mathrm{NE}$ & Lucena, MFA 1329 (ASE) \\
\hline \multicolumn{5}{|l|}{ Verbenaceae } \\
\hline Lantana camara L. & Arb & NR & $\mathrm{NE}$ & Viana, 1823 (ASE) \\
\hline Lantana fucata Lindl. & Arb & NR & $\mathrm{NE}$ & Almeida, C 10 (ASE) \\
\hline Lantana gracilis T.Silva & Arb & NR & RAR, NE & Dantas, TVP 14 (ASE) \\
\hline
\end{tabular}


Tabela 1. Continuação.

\begin{tabular}{|c|c|c|c|c|}
\hline Família/Espécie & HAB & OCO & CAT & Coletor no./Autor(es) \\
\hline Lantana lucida Schauer & Arb & - & NE, EMA & Matos, GMA 126 (ASE) \\
\hline Lantana radula $\mathrm{Sw}$. & Arb & NR & $\mathrm{NE}$ & Costa, SM 434 (ASE) \\
\hline Stachytarpheta angustifolia (Mill.) Vahl & Erv & - & $\mathrm{NE}$ & Santos, J s/n (ASE) \\
\hline Stachytarpheta cayennensis (Rich.) Vahl & Erv & - & $\mathrm{NE}$ & Vicente, A 121 (ASE) \\
\hline Tamonea sp. & Erv & - & $\mathrm{NE}$ & Viana, G 1829 (ASE) \\
\hline \multicolumn{5}{|l|}{ Violaceae } \\
\hline Pombalia calceolaria (L.) Paula-Souza & Erv & - & $\mathrm{NE}$ & Fonseca, MR 882 (ASE) \\
\hline \multicolumn{5}{|l|}{ Viscaceae } \\
\hline Phoradendron chrysocladon A.Gray & Erv-Hpa & - & NE & Santana, MC 141 (ASE) \\
\hline \multicolumn{5}{|l|}{ Vitaceae } \\
\hline Cissus erosa Rich. & Tre-L & - & $\mathrm{NE}$ & Silva, FO 200 (ASE) \\
\hline Cissus pinnatifolia Lombardi & Tre-L & - & $\begin{array}{c}\text { RAR, NE, } \\
\text { EMA }\end{array}$ & Ribeiro, A 526 (HUEFS) \\
\hline \multicolumn{5}{|l|}{ Vochysiaceae } \\
\hline Vochysia lucida C.Presl & Arv & - & $\mathrm{NE}$ & Araujo, D 1963 (ASE) \\
\hline \multicolumn{5}{|l|}{ Xyridaceae } \\
\hline Xyris anceps Lam. & Erv & - & $\mathrm{NE}$ & Santos, ACAS 38 (ASE) \\
\hline Xyris ciliata Thunb. & Erv & - & $\mathrm{NE}$ & Souza, CL 21 (ASE) \\
\hline Xyris fallax Malme & Erv & - & $\mathrm{NE}$ & Viana G, 418 (ASE) \\
\hline Xyris savanensis Miq. & Erv & - & $\mathrm{NE}$ & Costa, SM 309 (ASE) \\
\hline
\end{tabular}

As famílias de maior riqueza de Angiospermas foram: Fabaceae (73 espécies), Poaceae (65), Cyperaceae (59), Rubiaceae (40) e Asteraceae (36). As dez famílias de maior riqueza (Figura 3) somam $50.6 \%$ do total de espécies coletadas e $9.1 \%$ do total de famílias. Por sua vez, as famílias com apenas uma espécie representam $3 \%$ do total de espécies.

Em Sergipe, Fabaceae foi a família de maior riqueza em estudos realizados na Mata Atlântica (Mendes et al. 2010; Souza-Alves et al. 2014; Landim et al. 2015; Oliveira et al. 2016) e na Caatinga (Machado et al. 2012; Ferreira et al. 2013; Silva et al. 2013). No PARNA Serra de Itabaiana, sua importância já havia sido destacada para a fitofisionomia de Areias Brancas (Dantas et al. 2010). Ressalta-se que é uma das maiores famílias no Brasil, em que $67 \%$ das espécies são endêmicas (Giulietti et al. 2005). Ademais, é encontrada em diversos tipos de habitats, sendo parte desse sucesso atribuído à simbiose com bactérias fixadoras de nitrogênio, o que permite a estas espécies colonizar ambientes pobres desse macronutriente (Queiroz 2009).

Com relação ao número de espécies pertencentes à Poaceae, houve um significativo aumento em relação ao levantamento realizado por Maciel \& Alves (2011), que são especialistas nesta família e registraram 40 espécies e citaram pela primeira vez em Sergipe: Apochloa lutzii (Swallen) Zuloaga \& Morrone, Ichnanthus lancifolius Meze e Ocellochloa soderstromii (Zuloaga \& Send.) Zuloaga \& Morrone ex Filg \& Rodr. O gênero Paspalum L. está representado no estado por 19 espécies, sendo que 10 ocorrem no PARNA Serra de Itabaiana e destas, uma (Paspalum gardnerianum Nees) está registrada somente para esta localidade (Maciel 2013).

Na Flora de Sergipe foram registradas 101 espécies de Cyperaceae, distribuídas em 16 gêneros (Prata et al. 2013), já no presente estudo foram constatadas 59 espécies e 12 gêneros. Cyperaceae possui distribuição cosmopolita, frequente em locais abertos e encharcados (Souza \& Lorenzi 2012), fato que justifica a abrangência dessa família.

Já Orchidaceae no estado é representada por 68 espécies subordinadas a 36 gêneros (Monteiro et al. 2013), sendo 35 espécies e 18 gêneros registrados na área de estudo. Três espécies são restritas a essa unidade de conservação: Habenaria parviflora Lindl., Jacquiniella globosa (Jacq.) Schltr. e Liparis vexillifera (Lex.) Cogn.

Quanto ao hábito (Figura 4), houve um predomínio de ervas com 341 espécies (41.2\%), seguidas por arbustos com $204(24.7 \%)$ e árvores com 145 (17.6\%). Outros hábitos menos representativos foram: trepadeiras lenhosas $(6.3 \%)$, trepadeiras herbáceas $(3.5 \%)$, ervas epífitas $(3.4 \%)$ e o restante somaram $3.4 \%$. O aumento e a variedade no número de hábitos se deve ao englobamento das áreas de estudo por diversos pesquisadores. 


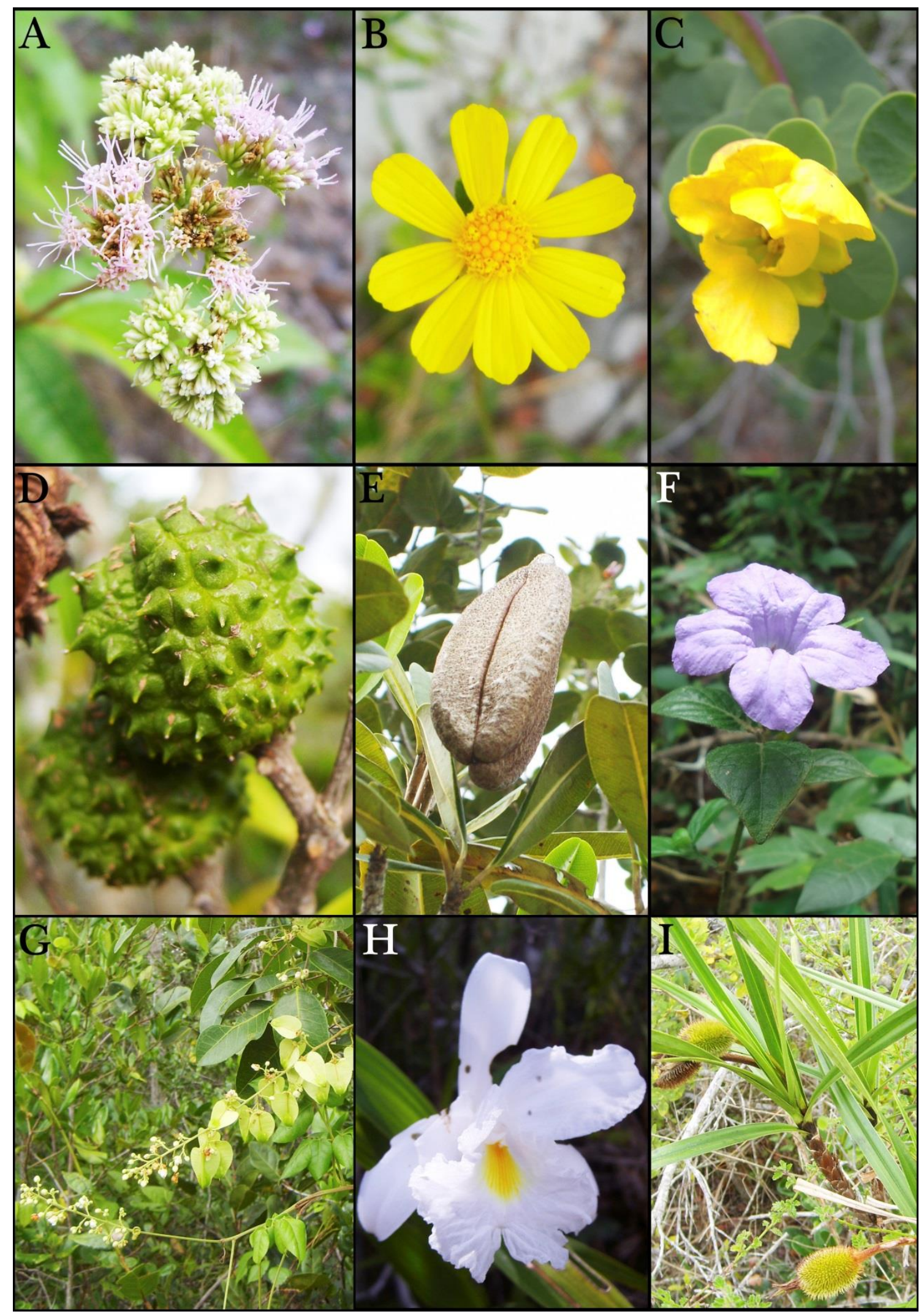

Figura 2. Alguns exemplos de espécies no Parque Nacional Serra de Itabaiana, Sergipe, Brasil: A. Acritopappus confertus (Gardner) R.M.King \& H.Rob.; B. Aspilia martii Baker; C. Chamaecrista cytisoides (DC. ex Collad.) H.S. Irwin \& Barneby; D. Esenbeckia grandiflora Mart.; E. Kielmeyera rugosa Choisy; F. Ruellia bahiensis (Nees) Morong; G. Serjania salzmanniana Schltdl.; H. Sobralia liliastrum Salzm. ex Lindl.; I. Vellozia dasypus Seub.). 


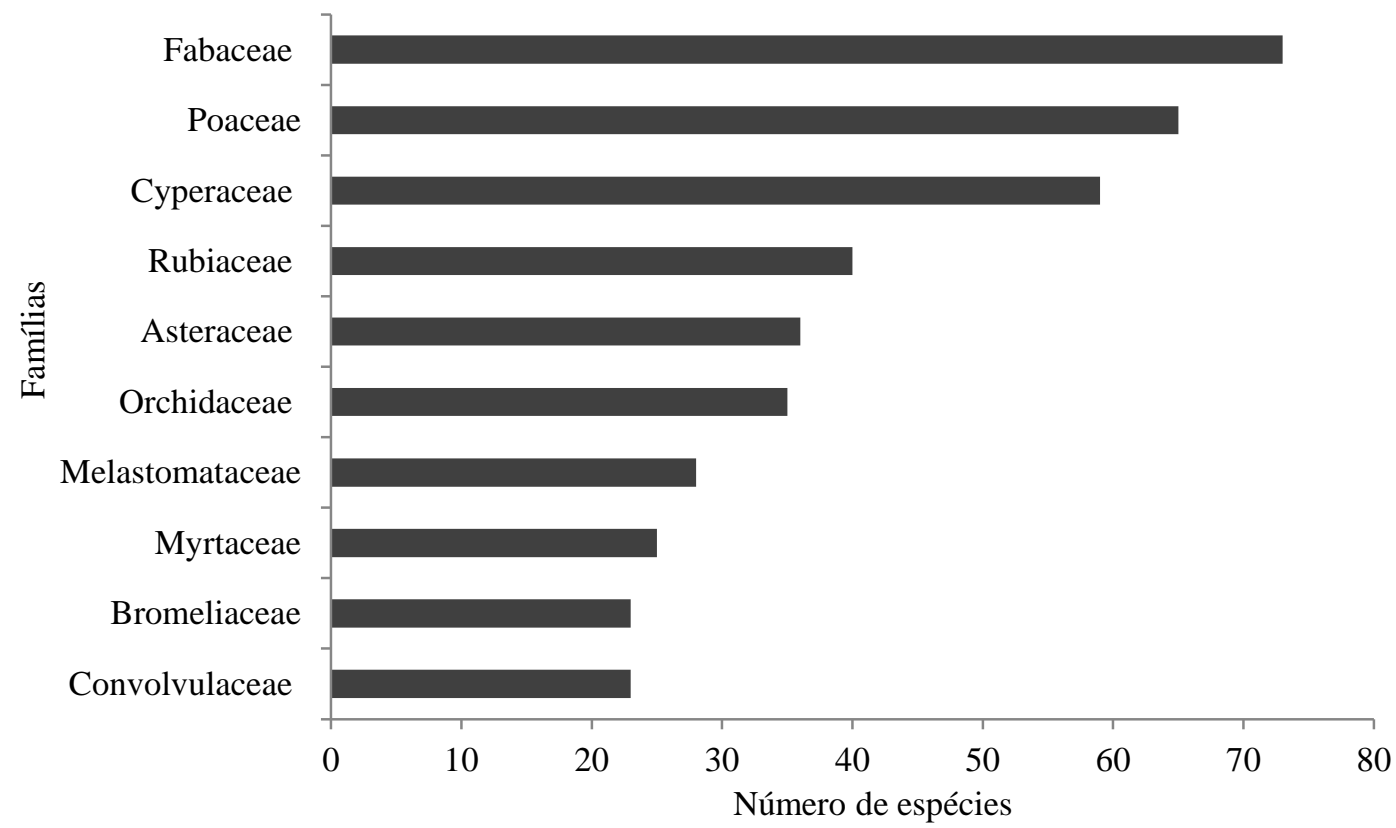

Figura 3. Famílias com maior riqueza de espécies no Parque Nacional Serra de Itabaiana, Sergipe, Brasil.

Com relação às ervas, destaca-se a nova ocorrência de Utricularia costata P.Taylor (Lentibulariaceae) para o nordeste (Carregosa \& Costa 2014), sendo que em Sergipe o atual registro foi apenas nesta Unidade de Conservação. As ervas epífitas estão representadas por 28 espécies, com maior número de representantes de Bromeliaceae e Orchidaceae, além de Araceae e Polypodiaceae. As ervas saprófitas foram representadas por espécies das famílias Burmanniaceae (Apteria aphylla (Nutt.) Barnhart ex Small e Burmannia capitata (Walter ex J.F. Gmel.) Mart.), Gentianaceae (Voyria obconica Progel) e Lauraceae (Cassytha filiformis L.).

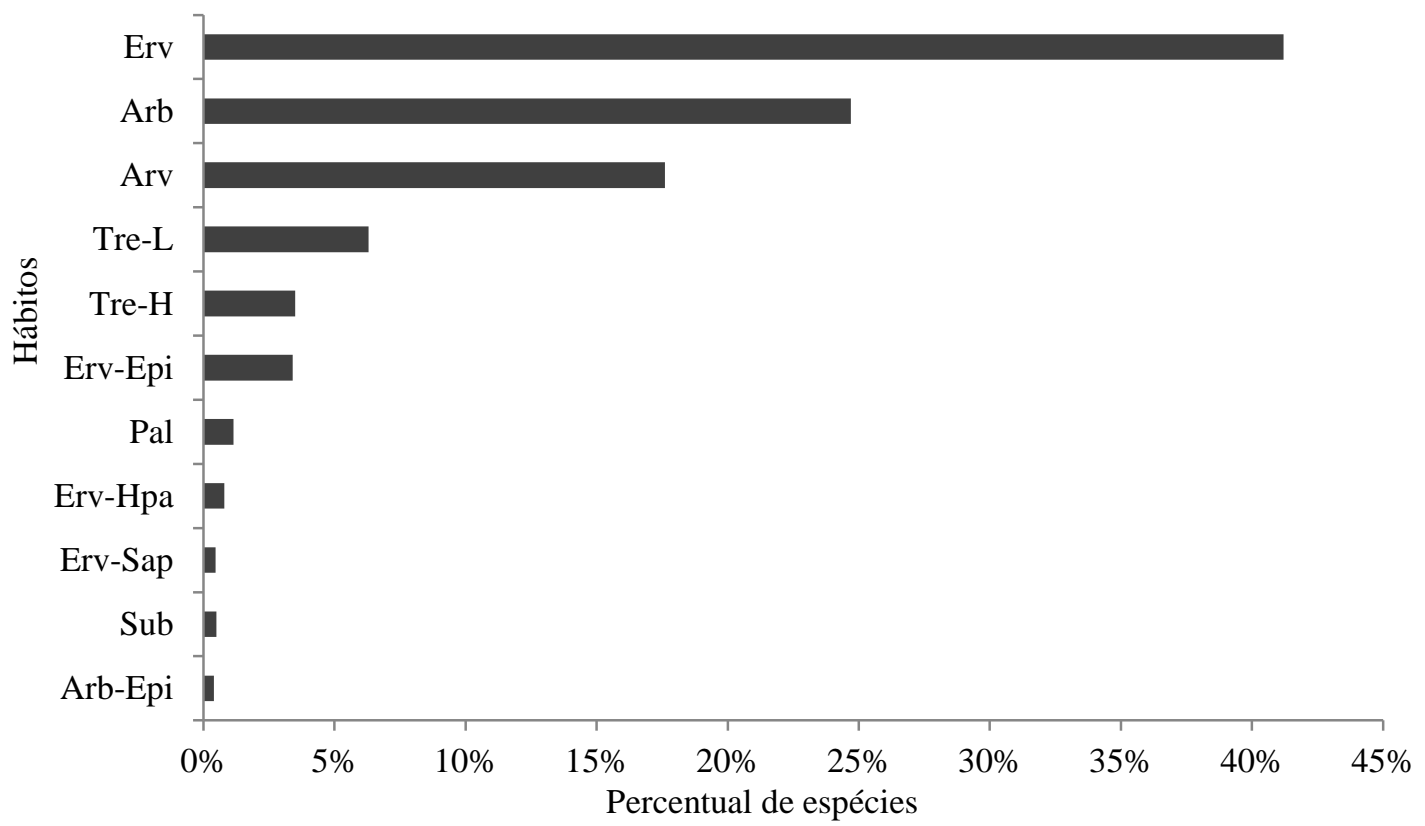

Figura 4. Riqueza de espécies por hábitos de plantas vasculares no Parque Nacional Serra de Itabaiana, Sergipe, Brasil (Erv: erva; Arb: arbusto; Arv: árvore; Tre-L: trepadeira lenhosa; Tre-H: trepadeira herbácea; Erv-Epi: erva epífita; Pal: palmeira; Arb-Hpa: arbusto hemiparasita; Erv-Sap: erva saprófita; Arb-Epi: arbusto epífita; Sub: subarbusto). 
No PARNA Serra de Itabaiana, muitos arbustos estão associados a ambientes de vegetação aberta com solo arenoso de forma agregada formando moitas. Nas Areias Brancas, as espécies: Agarista revoluta (Spreng.) J.D. Hook. ex Nied. (Ericaceae), Chamaecrista cytisoides (DC. ex Collad.) H.S. Irwin \& Barneby (Fabaceae), Coccoloba laevis Casar. (Polygonaceae) e Leptolobium bijugum (Spreng.) Vogel (Fabaceae) possuem maior importância fitossociológica (Dantas et al. 2010).

Foram registradas 170 novas ocorrências de espécies para o estado de Sergipe. Ressaltase que uma nova espécie para a ciência, que pertence à família Boraginaceae, foi descrita para o estado de Sergipe: Varronia johnstoniana J.I.M. Melo \& D.D.Vieira (Melo \& Vieira 2015). Já Aspilia itabaianensis J.U.Santos, ocorre exclusivamente em Sergipe, e mais especificamente, no PARNA Serra de Itabaiana.

Foram encontradas seis espécies consideradas raras: Chamaecrista cytisoides (DC. ex Collad.) H.S. Irwin \& Barneby (Fabaceae), Cissus pinnatifolia Lombardi (Vitaceae), Griffinia espiritensis Ravenna (Amaryllidaceae), Lantana gracilis T.Silva (Verbenaceae), Monteverdia opaca (Reissek) Biral (Celastraceae) e Spermacoce decipiens (K.Schum.) Kuntze (Rubiaceae).

De acordo com a categorias de espécies ameaçadas de extinção existem: três em perigo (Griffinia espiritensis Ravenna, Pouteria macahensis T.D. Penn. e Protium occhionii Rizzini), nove quase ameaçadas (por ex. Byrsonima bahiana W.R. Anderson e Catasetum uncatum Rolfe), três vulneráveis (Allagoptera brevicalyx M.Moraes, Cedrela odorata L. e Cryptanthus zonatus (Vis.) Bee), 69 menos preocupantes, duas com dados insuficientes (Aechmea lingulata (L.) Baker e Myrciaria tenella (DC.) O.Berg.) e a maioria das espécies não foi avaliada.

Foram catalogadas 80 (11.5\%) espécies endêmicas da Mata Atlântica (p. ex. Annona salzmannii A.DC., Blepharodon costae Fontella \& Morillo, Cryptanthus sergipensis I. Ramírez e Kielmeyera argentea Choisy) e 13 (0.6\%) endêmicas da Caatinga (p. ex. Croton grewioides Baill., Paspalum scutatum Nees ex Trin. e Ruellia asperula (Mart. ex Ness) Lindau). Destaca-se um número considerável de espécies da área de estudo que são comuns às áreas de Caatinga, apesar de não serem endêmicas deste bioma, o que justifica a classificação da área como ecótono (Mendes et al. 2010).

\section{Conclusão}

Este inventário florístico acrescenta novas ocorrências para o Parque Nacional (PARNA) Serra de Itabaiana e para o estado de Sergipe, amplia as informações sobre a distribuição de espécies e reflete o atual conhecimento nesta Unidade de Conservação. Reforça ser uma área de transição dos domínios fitogeográficos por conter espécies endêmicas da Mata Atlântica e da Caatinga. Ainda revela a existência de espécies raras, vulneráveis, quase ameaçadas, em perigo de extinção e de ocorrência restrita a essa área. Além disso, divulga a existência de uma nova espécie para a ciência. Esses fatos reforçam a importância biológica desse local e o seu papel na conservação da biodiversidade em Sergipe.

\section{Agradecimentos}

Conselho Nacional de Desenvolvimento Científico e Tecnológico (CNPq) - Projeto Flora de Sergipe (processo 472483/2011-0) e ao INCT - Herbário Virtual da Flora e dos Fungos.

\section{Referências}

APG IV - Angiosperm Phylogeny Group IV. (2016) An update of the Angiosperm Phylogeny Group Classification for the orders and families of flowering plants: APG IV. Botanical Journal of the Linnean Society, 181: 1-20. DOI: 10.1111/boj.12385

BFG - The Brazil Flora Group. (2018) Brazilian Flora 2020: Innovation and collaboration to meet Target 1 of the Global Strategy for Plant Conservation (GSPG). Rodriguésia, 69(4): 15131527. DOI: $10.1590 / 2175-7860201869402$ 
Brandon K., Fonseca G.A.B. da, Rylands A.B. \& Silva J.M.C. da. (2005) Conservação brasileira: desafios e oportunidades. Megadiversidade, 1(1): 7-13.

Brasil. Lei Federal No 9.985, de 18 de julho de 2000. Regulamenta o art. 225, § 1o, incisos I, II, III e VII da Constituição Federal, institui o Sistema Nacional de Unidades de Conservação da Natureza e dá outras providências. Disponível em: http://www.planalto.gov.br/ccivil_03/ leis/19 985.htm (Acessado em: 24/10/2018).

Brasil (2005) Decreto de 15-VI-2005. Criação do Parque Naciona Serra de Itabaiana. Brasília: Diário Oficial da União, Poder Executivo, 15-VI-2005.

Carregosa T. \& Costa S.M. (2014) Ampliação da distribuição geográfica de três espécies de Utricularia (Lentibulariaceae) para o bioma Mata Atlântica. Rodriguésia, 65(2): 563-565. DOI: 10.1590/S2175-78602014000200017

Carvalho G. (2017) Package "flora". Tools for Interacting with the Brazilian Flora 2020. Disponível em: https://cran.r-project.org/web/packages/flora/index.html (Acessado em 22/01/2019).

Christenhusz M.J.M. \& Byng J.W. (2016) The number of known plants species in the world and its annual increase. Phytotaxa, 261(3): 201-217. DOI: 10.11646/phytotaxa.261.3.1

Dantas T.V.P. \& Ribeiro A.S. (2010) Caracterização da vegetação do Parque Nacional Serra de Itabaiana, Sergipe - Brasil. Biotemas, 23(4): 9-18. DOI: 10.5007/2175-7925.2010v23n4p9

Dantas T.V.P., Nascimento-Júnior J.E., Ribeiro A.S. \& Prata A.P.N. (2010) Florística e estrutura da vegetação arbustivo-arbórea das Areias Brancas do Parque Nacional Serra de Itabaiana/Sergipe, Brasil. Revista Brasileira de Botânica, 33(4): 575-588. DOI: 10.1590/S010084042010000400006

Falcão V. (2004) Pinheiro do nordeste prestes a desaparecer. Ciência \& Meio Ambiente. Disponível em: https:/br.groups.yahoo.com/neo/groups/arvores/conversations/topics/4966 (Acessado em 23/10/2018).

Ferreira E.V.R., Prata A.P.N. \& Mello A.A. (2013) Floristic list from a Caatinga remnant in Poço Verde, Sergipe, Brazil. Check List, 9(6): 1354-1360. DOI: 10.15560/9.6.1354

Franco E. (1983) Biogeografia de Sergipe. Aracaju: Segrase. 136 p.

Giulietti A.M., Harley R.M., Queiroz L.P., Wanderley M.G.L. \& Berg C.V.D. (2005) Biodiversidade e conservação das plantas no Brasil. Megadiversidade, 1(1): 52-61.

Giulietti A.M., Rapini A., Andrade M.J.G., Queiroz L.P. \& Silva J.M.C. (2009) Plantas raras do Brasil. Belo Horizonte: Conservação Internacional \& Universidade Estadual de Feira de Santana. 496 p.

Ivanauskas N.M., Rodrigues R.R. \& Nave A.G. (1997) Aspectos ecológicos de um trecho de floresta de brejo em Itatinga, SP: florística, fitossociologia e seletividade de espécies. Revista Brasileira de Botânica, 20(2): 139-153. DOI: 10.1590/S0100-84041997000200005

Landim M.F., Proença E.C.B., Sales A.B. \& Matos I.S. (2015) Floristic characterization of an Atlantic Rainforest remnant in Southern Sergipe: Crasto Forest. Biota Neotropica, 15(1): 1-16. DOI: 10.1590/1676-06032014003613

Lima T.V. (2006) A distribuição eco-geográfica do Pinheiro Bravo no Brasil - Nordeste: um caso a parte. Nordeste rural - negócios do campo. Disponível em: http://www.nordesterural.com.br/n ordesterural/matler.asp?newsId=4107 (Acessado em 03/06/2014).

Lucena M.F.A., Amorim B.S. \& Alves M. (2009) Sinopse das espécies de Euphorbiaceae s.l. do Parque Nacional Serra de Itabaiana, Sergipe, Brasil. Caatinga, 22: 214-224.

Machado W.J., Prata A.P.N. \& Mello A.A. (2012) Floristic composition in areas of Caatinga and Brejo de Altitude in Sergipe state, Brazil. Checklist, 8(6): 1089-1101. DOI: 10.15560/8.6.1089

Maciel J.R. (2013) Poaceae I: Paspalum (p. 510-521). In: Prata A.P.N., Amaral, M.C.E., Farias M.C. \& Alves M.V. (Orgs). Flora de Sergipe. Volume 1. Aracaju: Gráfica e Editora Triunfo. 717 p.

Maciel J.R. \& Alves M. (2011) A família Poaceae na Serra de Itabaiana, Parque Nacional Serra de Itabaiana, Sergipe-Brasil. Revista Caatinga, 24(3): 85-93.

Melo J.I.M. \& Vieira D.D. (2015) A new species of Varronia (Cordiaceae) and a checklist of Boraginales for the State of Sergipe, Brazil. Phytotaxa, 231(2): 145-155. DOI: 10.11646/phytotaxa.231.2.3 
Mendes K., Gomes P. \& Alves M. (2010) Inventário florístico de uma área de tensão ecológica na Mata Atlântica do Nordeste do Brasil. Rodriguésia, 61(4): 669-676. DOI: 10.1590/21757860201061408

MMA - Ministério do Meio Ambiente (2003) Fragmentação de ecossistemas: causas, efeitos sobre a biodiversidade e recomendações de políticas públicas. Brasília: MMA/SBF. 508 p.

MMA - Ministério do Meio Ambiente (2007) Áreas prioritárias para conservação, uso sustentável e repartição dos benefícios da biodiversidade brasileira. Atualização - Portaria MMA n9, de 23 de janeiro de 2007. Brasília: Ministério do Meio Ambiente, Secretaria de Biodiversidade e Florestas.

Monteiro S.H.N., Carregosa T., Santos L.A.S. \& Matos I.S. (2013) Orchidaceae (p. 431-490). In: Prata A.P.N., Amaral M.C.E., Faria M.C. \& Alves M.V. (Orgs). Flora de Sergipe. Volume 1. Aracaju: Gráfica e Editora Triunfo. 717 p.

Oliveira I.S.S. (2008) Estudo dos impactos ambientais como subsídio para o planejamento dastrilhas do Parque Nacional na Serra de Itabaiana, SE. Boletim Goiano de Geografia, 28(1): 115-126. DOI: 10.5216/bgg.v28i1.4905

Oliveira E.V.S., Gomes L.A., Déda R.M., Melo L.M.S., Silva A.C.C., Farias M.C.V. \& Prata A.P.N. (2016) Floristic survey of the Mata do Junco Wildlife Refuge, Capela, Sergipe State, Brazil. Hoehnea, 43(4): 645-667. DOI: 10.1590/2236-8906-28/2016

Pereira A.F.N., Barros I.C.L., Santiago A.C.P. \& Silva I.A.A. (2011) Florística e distribuição geográfica das samambaias e licófitas da Reserva Ecológica de Gurjaú, Pernambuco, Brasil. Rodriguésia, 62(1): 1-10. DOI: 10.1590/2175-7860201162101

Pessoa E.M. \& Alves M. (2011) Orchidaceae Juss. na Serra de Itabaiana, Sergipe, Brasil. Revista Caatinga, 24(4): 102-114.

Prata A.P.N., Silva A.C., Lópes M.G., Costa S.M., Trevisan R., Ribeiro A.R.O., Alves M., Lemos Junior I.C. \& Nunes I.R. (2013) Cyperaceae (p. 127-218). In: Prata A.P.N., Amaral M.C.E., Farias M.C. \& Alves M.V. (Orgs). Flora de Sergipe. Volume 1. Aracaju: Gráfica e Editora Triunfo. $717 \mathrm{p}$.

Primack R. \& Rodrigues E. (2001) Biologia da Conservação. Londrina: E. Rodrigues. 328 p.

Queiroz L.P. (2009) Leguminosas da Caatinga. Feira de Santana: Universidade Estadual de Feira de Santana. $913 \mathrm{p}$.

R Development Core Team (2015) R: A language and environment for statistical computing. Disponível em: http://www.R-project.orgS (Acessado em 12/10/2018).

Santos A.F. \& Andrade J.A. (1992) Delimitação e regionalização do Brasil Semiárido: Sergipe. Aracaju: Universidade Federal de Sergipe. 72 p.

Seplan (1979) Atlas de Sergipe. Aracaju: Universidade Federal de Sergipe, Secretaria do Planejamento do Estado de Sergipe. 95 p.

Silva A.C.C., Prata A.P.N. \& Mello A.A. (2013) Flowering plants of the Grota do Angico Natural Monument, Caatinga of Sergipe, Brazil. Check List, 9(4): 733-739.

Sobral I.S., Santana R.K.O., Gomes L.J., Costa M., Ribeiro G.T. \& Santos J.R. (2007) Avaliação dos impactos ambientais no Parque Nacional Serra de Itabaiana - SE. Caminhos de Geografia, 8(24): 102-110.

Souza V.C. \& Lorenzi H. (2012) Botânica Sistemática: guia ilustrado para identificação das famílias de Fanerógamas nativas e exóticas no Brasil, baseado em APG III. $3^{\circ}$ edição. São Paulo, Nova Odessa: Instituto Plantarum. 768 p.

Souza-Alves J.P., Barbosa M.R.V., Ferrari S.F. \& Thomas W.W. (2014) Diversity of trees and lianas in two sites in the Coastal Atlantic Forest of Sergipe, Northeastern Brazil. Checklist, 10(4): 709-717.

Stehmann J.R., Forzza R.C., Salino A., Sobral M., Costa D.P. \& Kamino L.H.Y. (Eds) (2009) Plantas da Floresta Atlântica. Rio de Janeiro: Jardim Botânico do Rio de Janeiro. 516 p.

Vicente A. (1999) Levantamento florístico de um fragmento florestal na Serra de ItabaianaSergipe. Dissertação de Mestrado, Programa de Pós-Graduação em Biologia Vegetal. Universidade Federal de Pernambuco, Recife. 
Vicente A., Ribeiro A.S., Santos E.A. \& Franco C.R.P. (2005) Levantamento Botânico (p. 15-37). In: Carvalho C.M. \& Vilar J.C. (Coords). Parque Nacional Serra de Itabaiana - Levantamento da Biota. Aracaju: Ibama, Biologia Geral e Experimental-UFS. 131 p. 